\title{
Cooperative Constrained Control of Distributed Agents with Nonlinear Dynamics and Delayed Information Exchange: a Stabilizing Receding-Horizon Approach
}

\author{
Elisa Franco, Lalo Magni, Thomas Parisini, Marios M. Polycarpou, \\ and Davide M. Raimondo
}

\begin{abstract}
This paper addresses the problem of cooperative control of a team of distributed agents with decoupled nonlinear discrete-time dynamics, which operate in a common environment and exchange delayed information between them. Each agent is assumed to evolve in discrete-time, based on locally computed control laws, which are computed by exchanging delayed state information with a subset of neighboring agents. The cooperative control problem is formulated in a receding-horizon framework, where the control laws depend on the local state variables (feedback action) and on delayed information gathered from cooperating neighboring agents (feedforward action). A rigorous stability analysis exploiting the input-to-state stability properties of the receding-horizon local control laws is carried out. The stability of the team of agents is then proved by utilizing smallgain theorem results.
\end{abstract}

\section{INTRODUCTION}

$\mathbf{T}$ He design and analysis of decentralized control systems have been under research investigation for more than thirty years. Many problems falling into this category have been addressed with various mathematical tools, while new theoretical and application issues are arising as a result of current trends in distributed systems, such as the increasing size and complexity of feedback control systems, the availability of spatially distributed sensors and actuators, and the need to come up with more autonomous systems.

When dealing with large scale systems, one of key objectives is to find conditions guaranteeing closed-loop stability, while reducing the computational load stemming from a centralized approach. Starting with the notion of "fixed modes" introduced in the 1970s for linear large scale systems [1], other investigations focused on the structure and size of interconnections [2]. Following the reasoning of this latter aspect, adaptive control [3], and more recently model predictive

Corresponding author: Thomas Parisini. This work has been partially supported by the Italian Ministry for University and Research and by the University of Cyprus Research Fund.

E. Franco is with the Dept. of Control and Dynamical Systems, California Institute of Technology, USA. (elisaf@caltech.edu)

L. Magni and D. M. Raimondo are with the Dept. of Computer and Systems Sciences, University of Pavia, Italy. ( $\{$ lalo.magni,davide.raimondo\}@unipv.it)

T. Parisini is with the Dept. of Electrical, Electronic and Computer Engineering, DEEI-University of Trieste, Italy. (parisini@units.it)

M. M. Polycarpou is with the Dept. of Electrical and Computer Engineering, University of Cyprus, Nicosia 1678, Cyprus. (mpolycar@ucy.ac.cy) control [4] approaches have been proposed. Specific emphasis on the structural properties of decentralized controlled largescale systems is given in the research work of D'Andrea and co-workers (see, for instance, [5]), which is used in several applications, such as flight formation and distributed sensors. Studies on topology independent control have also been recently reported [6].

Another related research direction in decentralized control considers the problem of controlling a team of dynamically decoupled cooperating systems. For instance, there have been some important theoretical results on the stability of swarms [7], but a considerable number of publications in this area focus on specific issues related to Uninhabited Autonomous/Air Vehicles (UAVs) applications (see, for instance, [8], [9], [10]).

One of the approaches used in this area is based on the selection of a suitable cost function and its optimization in a model-predictive control (MPC) framework. MPC is nowadays a very active research area [11], [12], [13], [14], [15], [16], [17]. A good survey on this topic can be found in [18].

The cost function used for MPC framework can take into account several issues, such as collision avoidance and formation constraints, and may reward the tracking of a certain path. In [19], [20] and [15], the authors consider a two-degrees of freedom team of UAVs assigned to visit a certain number of points. The team of UAVs is controlled in a centralized receding-horizon $(\mathrm{RH})$ framework and by exploiting global potential functions, the authors prove certain stationarity properties of the generated trajectories in the case of two agents searching for multiple targets. A RH control scheme has also been proposed in [21], [22], where a centralized problem is decomposed to allow local computations and feasibility issues are thoroughly examined; stability is obtained in [21] exploiting a hierarchical decomposition of the team in suitable subgraphs with assigned priorities.

Coordination of a large group of cooperating nonlinear vehicles is considered in [23] and related works, where a centralized RH problem is decomposed and solved locally. Convergence to the formation equilibrium point is assured by guaranteeing frequent updates and a bounded error between the assumed and the predicted trajectories, which every agent computes for itself and it's neighbors in the model predictive control process.

Towards a broad analysis of the structural properties of 
cooperative systems, an Input-to-State Stability (ISS) analysis has recently been proposed by several authors. In [24], [25] the concept of Leader to Formation Stability is developed. A discussion of some of the issues arising in the study of non-holonomic vehicles using ISS can be found in [26]. ISS tools have been successfully applied to the specific case of networked systems with serial communication, where Nesic and Teel propose a new unified framework for modelling and analyzing networked control systems [27], [28]. Finally, in [13], [17] connections are made between ISS and MPC.

In this paper we consider a cooperative control problem for a team of distributed agents with nonlinear discrete-time dynamics. The problem formulation is based on a completely decentralized RH control algorithm, which is analyzed using an ISS approach. The proposed scheme generalizes the approach presented in [29] to a nonlinear framework. Each agent is assumed to evolve in discrete-time by means of locally computed control laws, which takes into consideration delayed state information from a subset of neighboring cooperating agents. The cooperative control problem is first formulated in a RH framework, where the control laws depend on the local state variables (feedback action) and on delayed information gathered from cooperating neighboring agents (feedforward action). A rigorous stability analysis is carried out, exploiting the ISS properties of the RH local control laws. The asymptotic stability of the team of agents is then proved by utilizing small-gain theorem results. The information flow among the agents is considered as a set of interconnections whose size is measured by the weight this information has in the computation of the control action. Hence, the derived result confirms that, in this framework, a suitable "interconnection" boundedness is necessary to guarantee stability.

The paper is organized as follows. Section II introduces the main notations and basic definitions. Section III formulates the multi-agent cooperative control problem in a discrete-time RH framework. The general stability proofs are presented and discussed in Section IV and in the Appendix. Some simulation results are presented in Section V. The conclusions are reported in Section VI.

\section{MAin NOTATIONS AND BASIC DEFINITIONS}

This section provides the main notations and definitions ${ }^{1}$ used in the paper. Let $\mathbb{R}, \mathbb{R}_{\geq 0}, \mathbb{Z}$, and $\mathbb{Z}_{\geq 0}$ denote the real, the non-negative real, the integer, and the non-negative integer sets of numbers, respectively. The Euclidean norm is denoted as $|\cdot|$. For any discrete-time sequence $\phi: \mathbb{Z}_{\geq 0} \rightarrow$ $\mathbb{R}^{m},\|\phi\| \triangleq \sup _{k \geq 0}\left\{\left|\phi_{k}\right|\right\}$ and $\left\|\phi_{[\tau]}\right\| \triangleq \sup _{0 \leq k \leq \tau}\left\{\left|\phi_{k}\right|\right\}$, where $\phi_{k}$ denotes the value that the sequence $\phi$ takes in correspondence to the index $k$. The set of discrete-time sequences $\psi$ taking values in some subset $\Psi \subset \mathbb{R}^{m}$ is denoted by $\mathcal{M}_{\Psi}$. The symbol Id represents the identity function from $\mathbb{R}$ to $\mathbb{R}$, while $\gamma_{1} \circ \gamma_{2}$ is the composition of two functions $\gamma_{1}$ and $\gamma_{2}$ from $\mathbb{R}$ to $\mathbb{R}$. Given a set $A \subseteq \mathbb{R}^{n}, d(\zeta, A) \triangleq \inf \{|\eta-\zeta|, \eta \in A\}$ is the point-to-set distance from $\zeta \in \mathbb{R}^{n}$ to $A$. The difference between two given sets $A \subseteq \mathbb{R}^{n}$ and $B \subseteq \mathbb{R}^{n}$, with $B \subseteq A$,

\footnotetext{
${ }^{1}$ The notations and definitions introduced in the paper are fairly standard in the literature (see, for instance, [30]).
}

is denoted as $A \backslash B \triangleq\{x: x \in A, x \notin B\}$. Given a closed set $A \subset \mathbb{R}^{n}, \partial A$ denotes the boundary of $A$.

Definition 2.1 ( $\mathcal{K}$-function): A function $\gamma: \mathbb{R}_{\geq 0} \rightarrow \mathbb{R}_{\geq 0}$ is of class $\mathcal{K}$ (or a " $\mathcal{K}$-function") if it is continuous, positive definite and strictly increasing.

Definition $2.2\left(\mathcal{K}_{\infty}\right.$-function): A function $\gamma: \mathbb{R}_{\geq 0} \rightarrow \mathbb{R}_{\geq 0}$ is of class $\mathcal{K}_{\infty}$ if it is a $\mathcal{K}$-function and $\gamma(s) \rightarrow+\infty$ as $s \rightarrow+\infty$.

Definition 2.3 ( $\mathcal{K} \mathcal{L}$-function): A function $\beta: \mathbb{R}_{\geq_{0}} \times \mathbb{Z}_{\geq_{0}} \rightarrow$ $\mathbb{R}_{\geq_{0}}$ is of class $\mathcal{K} \mathcal{L}$ if, for each fixed $t \geq 0, \beta(\cdot, t)$ is of class $\mathcal{K}$, for each fixed $s \geq 0, \beta(s, \cdot)$ is decreasing and $\beta(s, t) \rightarrow 0$ as $t \rightarrow \infty$.

Definition 2.4 (Upper limit): Given a bounded sequence $s$ : $\mathbb{Z}_{\geq 0} \rightarrow \mathbb{R}_{\geq 0}$, the upper limit is defined as

$$
\varlimsup_{t \rightarrow \infty} s_{t} \triangleq \inf _{t \geq 0} \sup _{\tau \geq t} s_{\tau}
$$

Consider the following nonlinear discrete-time dynamic system

$$
x_{t+1}=\tilde{f}\left(x_{t}, w_{t}\right), t \geq 0, x_{0}=\bar{x},
$$

where $\tilde{f}(0,0)=0$, and where $x_{t} \in \mathbb{R}^{n}$ and $w_{t} \in W \subset \mathbb{R}^{r}$ are the state and the bounded input of the system, respectively. The discrete-time state trajectory of the system (1) with initial state $\bar{x}$ and input sequence $w=\left\{w_{t}, t \geq 0\right\}$ is denoted by $x(t, \bar{x}, w), t \geq 0$. We have the following further definitions.

Definition 2.5 (Robust positively invariant set): A set $\Xi \subset$ $\mathbb{R}^{n}$ is a robust positively invariant set for system (1) if $\tilde{f}(x, w) \in \Xi, \forall x \in \Xi$ and $\forall w \in W$.

Definition 2.6 (0-AS in $\Xi)$ : Given a compact set $\Xi \subset \mathbb{R}^{n}$ including the origin as an interior point, the system (1) with $w_{t}=0, \forall t \geq 0$ is said to be $0-A S$ (zero-asymptotically stable) in $\Xi$, if $\Xi$ is positively invariant for (1) and if there exists a $\mathcal{K} \mathcal{L}$-function $\beta$ such that

$$
|x(t, \bar{x}, 0)| \leq \beta(|\bar{x}|, t), \forall t \geq 0, \forall \bar{x} \in \Xi .
$$

Definition 2.7 (Regional ISS in $\Xi$ ): Given a compact set $\Xi \subset \mathbb{R}^{n}$ including the origin as an interior point, the system (1) with $w \in \mathcal{M}_{W}$, is said to be regionally ISS in $\Xi$, if $\Xi$ is robust positively invariant for (1) and if there exist a $\mathcal{K} \mathcal{L}$ function $\beta$ and a $\mathcal{K}$-function $\gamma$ such that

$$
|x(t, \bar{x}, w)| \leq \beta(|\bar{x}|, t)+\gamma(\|w\|), \forall t \geq 0, \forall \bar{x} \in \Xi .
$$

Definition 2.8 ( $A G$ in $\Xi$ ): Given a compact set $\Xi \subset \mathbb{R}^{n}$ including the origin as an interior point, the system (1) is said to have the Asymptotic Gain $(A G)$ property in $\Xi$, if $\Xi$ is robust positively invariant for (1) and if there exists a $\mathcal{K}_{\infty}$ function 
$\gamma_{A G}$ such that, for all initial state vectors $\bar{x} \in \Xi$ and all input sequences $w \in \mathcal{M}_{W}$, we have

$$
\varlimsup_{t \rightarrow \infty}|x(t, \bar{x}, w)| \leq \gamma_{A G}(\|w\|) .
$$

Definition $2.9(L S)$ : The system (1) satisfies the Local Stability $(L S)$ property, if for each $\varepsilon>0$, there exists a $\delta>0$ such that

$$
|x(t, \bar{x}, w)| \leq \varepsilon, \forall t \geq 0,
$$

for all $|\bar{x}| \leq \delta$ and all $\|w\| \leq \delta$.

Definition 2.10 ( $U A G$ in $\Xi$ ): Given a compact set $\Xi \subset \mathcal{R}^{n}$ including the origin as an interior point, the system (1) with $w \in \mathcal{M}_{W}$ satisfies the Uniform Asymptotic Gain (UAG) property in $\Xi$, if $\Xi$ is robust positively invariant for (1) and if there exists a $\mathcal{K}$-function $\gamma$ such that for each $\varepsilon>0$ and $\nu>0, \exists T=T(\varepsilon, \nu)$ such that

$$
|x(k, \bar{x}, w)| \leq \gamma(\|w\|)+\varepsilon,
$$

for all $\bar{x} \in \Xi$ with $|\bar{x}| \leq \nu$, and all $k \geq T$.

In the next section, the cooperative control problem addressed in the paper will be formulated in general terms, whereas, in Section IV, the stability properties of the distributed controlled system will be analyzed and the main results will be proved.

\section{PROBLEM FORMULATION}

We consider a distributed dynamic system made of a set of $M$ agents denoted as $\mathcal{A} \triangleq\left\{\mathcal{A}^{i}: i=1, \ldots, M\right\}$. Each agent $\mathcal{A}^{i}$ is described by the nonlinear time-invariant state equation:

$$
x_{t+1}^{i}=f^{i}\left(x_{t}^{i}, u_{t}^{i}\right), t \geq 0, x_{0}^{i}=\bar{x}^{i},
$$

where, for each $i=1, \ldots, M, x_{t}^{i} \in \mathbb{R}^{n^{i}}$ denotes the local state vector and $u_{t}^{i} \in \mathbb{R}^{m^{i}}$ denotes the local control vector of agent $\mathcal{A}^{i}$ at time $t$, and where we assume that $f^{i}(0,0)=$ $0, i=1, \ldots, M$. We also suppose that the dynamics of all $M$ agents evolve on the same discrete-time space (that is, the agents are synchronized).

The state vector $x_{t}^{i}$ of each agent $\mathcal{A}^{i}: i=1, \ldots, M$ is constrained to belong to a compact set $X^{i}$, that is,

$$
x_{t}^{i} \in X^{i} \subset \mathbb{R}^{n^{i}} .
$$

Analogously, the control vector $u_{t}^{i}$ is constrained to take values in a compact set $U^{i}$, that is,

$$
u_{t}^{i} \in U^{i} \subset \mathbb{R}^{m^{i}} .
$$

In open-loop mode, each agent is dynamically decoupled from the remaining agents and the dynamics of the other agents are not assumed to be known. The coupling between agents arises due to the fact that they operate in the same environment and due to the "cooperative" objective imposed on each agent by a cost function defined later on.

To achieve some degree of cooperation, each agent $\mathcal{A}^{i}$ exchanges an information vector $w_{t}^{i}$ with a given set of neighboring agents $\mathcal{G}^{i} \triangleq\left\{\mathcal{A}^{j}: j \in G^{i}\right\}$, where $G^{i}$ denotes the set of indexes identifying the agents belonging to the set $\mathcal{G}^{i}$. More precisely, the information exchange pattern is defined as follows. Let us consider a generic time-instant $t$; then for each $i=1, \ldots, M$, the agent $\mathcal{A}^{i}$ receives from each neighboring cooperating agent $\mathcal{A}^{j} \in \mathcal{G}^{i}$ the value of its local state vector with a delay of $\Delta_{i j}$ time steps, that is, agent $\mathcal{A}^{i}$ receives the vector $x_{t-\Delta_{i j}}^{j}$ from agent $\mathcal{A}^{j} \in \mathcal{G}^{i}$. To gain some more insight into the information exchange pattern, refer to Fig. 1, where a simple three-agent example is shown pictorially. In this specific example, each agent receives information from all remaining agents. At each time-instant $t$, we group all inputs to agent $\mathcal{A}^{i}$ into a vector $\bar{w}_{t}^{i}$ defined as $\bar{w}_{t}^{i} \triangleq \operatorname{col}\left(x_{t-\Delta_{i j}}^{j}, j \in G^{i}\right)$. The size of vector $\bar{w}^{i}$ is equal to $n_{w}^{i}=\sum_{j \in G^{i}} n^{j}$ and clearly

$$
\bar{w}_{t}^{i} \in W^{i},
$$

where $W^{i}$ denotes the cartesian product of all sets $X^{j}, j \in$ $G^{i}$, that is, $W^{i} \triangleq \prod_{j \in G^{i}} X^{j}$.

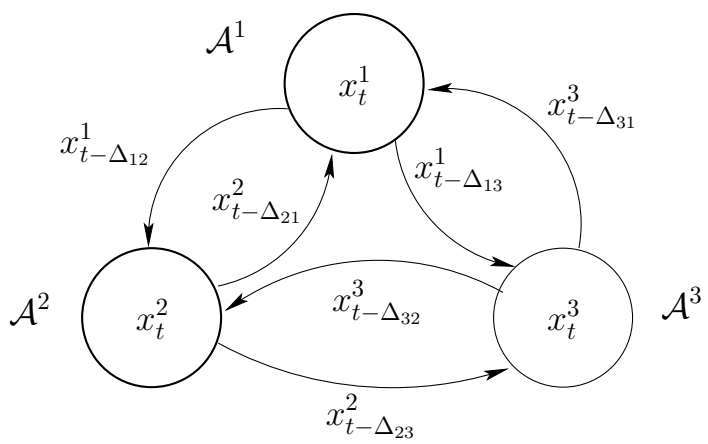

Fig. 1. Three agents exchanging delayed state information.

It is worth noting that the above setting allows the investigation of quite a large class of distributed cooperating dynamic systems like teams of mobile vehicles, cooperating robotic arms, routing nodes in communications and/or transportation networks where agents cooperate to minimize the total traffic delay, networks of reservoirs in water-distribution networks, etc..

For each $i=1, \ldots, M$ and for given values of the state vector $x_{t}^{i} \in X^{i}$ and of the information vector $\bar{w}_{t}^{i} \in W^{i}$ at time-instant $t$, we now introduce the following finite-horizon (FH) cost function (in general, nonquadratic):

$$
\begin{aligned}
J_{F H}^{i}\left(x_{t}^{i},\right. & \left.w_{t}^{i}, d_{t, t+N_{p}^{i}}^{h^{i}}, d_{t, t+N_{p}^{i}-1}^{q^{i}}, u_{t, t+N_{c}^{i}-1}^{i}, N_{c}^{i}, N_{p}^{i}\right) \\
& =\sum_{l=t}^{t+N_{p}^{i}-1}\left[h^{i}\left(x_{l}^{i}, u_{l}^{i}, d_{l}^{h^{i}}\right)+q^{i}\left(x_{l}^{i}, w_{l}^{i}, d_{l}^{q^{i}}\right)\right] \\
& +h_{f}^{i}\left(x_{t+N_{p}^{i}}^{i}, d_{t+N_{p}^{i}}^{h^{i}}\right),
\end{aligned}
$$

where, for a generic vector $r_{t}$, we define $r_{t, \tau} \triangleq$ $\operatorname{col}\left(r_{t}, \ldots, r_{\tau}\right)$ for both finite and infinite values of $\tau$. The positive integers $N_{c}^{i}$ and $N_{p}^{i}, i=1, \ldots, M$ denote the lengths of the so-called control and prediction horizons, respectively, according to the framework proposed in [31]. The 
local cost function is composed of two terms: a partial cost term given by $\sum_{l=t}^{t+N_{p}^{i}-1} h^{i}\left(x_{l}^{i}, u_{l}^{i}, d_{l}^{h^{i}}\right)+h_{f}^{i}\left(x_{t+N_{p}^{i}}^{i}, d_{t+N_{p}^{i}}^{h^{i}}\right)$, where $h^{i}$ is a transition cost function and $h_{f}^{i}$ is a terminal cost function, and a "cooperation" cost term given by $t+N_{p}^{i}-1$

$\sum_{l=t}^{p} q^{i}\left(x_{l}^{i}, w_{l}^{i}, d_{l}^{q^{i}}\right) ;$ the quantities $d_{l}^{h^{i}}, d_{l}^{q^{i}}, i=1, \ldots, M$ denote some given vectors of appropriate dimensions. In general, the vectors $d_{l}^{h^{i}}$ are useful to specify some reference value for some or all components of the local state variables, whereas the vectors $d_{l}^{q^{i}}$ can be used to parametrize the cooperation between the agents. For example (see also Section V), if the agents represent UAV vehicles, then vectors $d_{l}^{h^{i}}, d_{l}^{q^{i}}$ could be defined so as to specify given trajectories to be followed by each agent and also given "formation structures" for the agents. As will be subsequently clarified, the control variables $u_{l}^{i}, l=t, \ldots, t+N_{c}^{i}-1$ will be the argument of a suitable optimization problem, whereas the control variables $u_{l}^{i}, l=$ $t+N_{c}^{i}, \ldots, t+N_{p}^{i}-1$ will be obtained through some auxiliary control law $u_{l}^{i}=\kappa_{f}^{i}\left(x_{l}^{i}\right)$. The vector $w_{l}^{i}$ denotes the state of the dynamic system

$$
w_{l+1}^{i}=A_{w}^{i} w_{l}^{i}, l=t, \ldots, t+N_{p}^{i}-2 ; w_{t}^{i} \triangleq \bar{w}_{t}^{i},
$$

where $A_{w}^{i} \triangleq \alpha_{w}^{i} I_{n_{w}^{i}}$ with $\alpha_{w}^{i}<1$ and with $I_{n_{w}^{i}}$ denoting the identity matrix of dimension $n_{w}^{i}$. The dynamic system (8) is introduced in order to decrease the "importance" of the information vector in the $\mathrm{FH}$ cost function along the prediction horizon (e.g., a "forward-forgetting-factor" is introduced in the cost function as regards the information vector exchanged at time-instant $t$ ). It is worth noting that, at time-instant $t$, vectors $w_{l}^{i}$ can be considered as known external inputs in the cost function.

In the paper, for the sake of simplicity, we suppose that, by a suitable change of state coordinates, it is possible to consider an equivalent formulation where the cost function (with straightforward re-definitions of the symbols) can be rewritten in the simpler form

$$
\begin{aligned}
& J_{F H}^{i}\left(x_{t}^{i}, w_{t}^{i}, u_{t, t+N_{c}^{i}-1}^{i}, N_{c}^{i}, N_{p}^{i}\right) \\
& =\sum_{l=t}^{t+N_{p}^{i}-1}\left[h^{i}\left(x_{l}^{i}, u_{l}^{i}\right)+q^{i}\left(x_{l}^{i}, w_{l}^{i}\right)\right]+h_{f}^{i}\left(x_{t+N_{p}^{i}}^{i}\right),
\end{aligned}
$$

where $h^{i}(0,0)=0, q^{i}(0,0)=0$, and $h_{f}^{i}(0)=0$. Moreover, the origin is an interior point of the sets $X^{i}$ and $U^{i}$.

Remark 3.1: Beyond allowing for a simpler problem formulation, the reduction of the original $\mathrm{FH}$ cost function to the form (9) will allow for the design of time-invariant control laws; after a change of coordinates it will also be possible to carry on the stability analysis with reference to the origin as equilibrium state of the time-invariant system (see Section V for some details about the above change of coordinates in a practical simple case). However, considering the general case would not involve major conceptual difficulties.

The local control law is designed according to a RH strategy.
In the literature several different problem formulations can be found depending on the particular setting. In this paper, we state the RH control problem according to [31] (see also the well-known survey paper [18]).

Problem 3.1 (FH Optimal Control Problem): At every time instant $t \geq 0$, for every agent $\mathcal{A}^{i}, i=1, \ldots, M$ described by (4), for given control and prediction horizons lengths $N_{c}^{i}$ and $N_{p}^{i}$, for given auxiliary control laws $\kappa_{f}^{i}$, for given transition, cooperation and terminal cost functions $h^{i}, q^{i}, h_{f}^{i}$, for given terminal sets $X_{f}^{i}$, and for given values $x_{t}^{i} \in X^{i}$ and $\bar{w}_{t}^{i} \in W^{i}$ of the state and the information vectors, find the optimal $\mathrm{FH}$ control sequence $\left\{u_{t}^{i^{F H^{\circ}}}, \ldots, u_{t+N_{c}^{i}-1}^{i^{F H^{\circ}}}\right\}$ that minimizes cost (9) subject to:

1) the agent's dynamics (4) with $x_{t}^{i}$ as initial state and the vectors $w_{l}^{i}, l=t, \ldots, t+N_{p}^{i}-1$ given by (8) with $\bar{w}_{t}^{i}$ as initial condition;

2) the auxiliary control law $u_{l}^{i}=\kappa_{f}^{i}\left(x_{l}^{i}\right), l=t+$ $N_{c}^{i}, \ldots, t+N_{p}^{i}-1$;

3) the constraints (5) and (6), that is $x_{l}^{i} \in X^{i}, u_{l}^{i} \in U^{i}, l=$ $t, \ldots, t+N_{p}^{i}-1$

4) the terminal state constraint $x_{t+N_{p}^{i}}^{i} \in X_{f}^{i}$.

Clearly, by definition, the optimal FH control sequence $\left\{u_{t}^{i^{F H^{\circ}}}, \ldots, u_{t+N^{i}-1}^{i^{F H^{\circ}}}\right\}$ solving Problem 3.1 is such that, when applied to (4), the constraints (5), (6), and the terminal constraint $x_{t+N_{p}^{i}}^{i} \in X_{f}^{i}$ are simultaneously satisfied. Indeed, the following definition regarding a generic control sequence $u_{t, t+N_{c}^{i}-1}^{i}$ will be useful in the analysis reported in Section IV.

Definition 3.1 (Admissible control sequence): Given an initial state $x_{t}^{i}$, the sequence $u_{t, t+N_{c}^{i}-1}^{i}$ is said to be an admissible control sequence for the FH optimal control Problem 3.1 if its application to (4) under the action of the auxiliary control law $u_{l}^{i}=\kappa_{f}^{i}\left(x_{l}^{i}\right), l=t+N_{c}^{i}, \ldots, t+N_{p}^{i}-1$ allows simultaneous satisfaction of (5), (6) and of the terminal constraint $x_{t+N_{p}^{i}}^{i} \in X_{f}^{i}$.

Now, the $\mathrm{RH}$ procedure can be described in the usual way as follows. When the controlled agent $\mathcal{A}^{i}$ is in the state $x_{t}^{i}$ at stage $t$, the $\mathrm{FH}$ optimal control Problem 3.1 is solved, thus obtaining the sequence of optimal control vectors, $\left\{u_{t}^{i^{F H^{\circ}}}, \ldots, u_{t+N_{c}^{i}-1}^{i^{F H^{\circ}}}\right\}$. The first control action of this sequence becomes the control action $u_{t}^{i^{R H^{\circ}}}$ generated by the RH local controller at time-instant $t$ (i.e., $u_{t}^{i^{R H^{\circ}}} \triangleq$ $\left.u_{t}^{i^{F H^{\circ}}}\right)$. This procedure is repeated stage after stage and a feedback-feedforward control law $\gamma_{R H^{\circ}}^{i}\left(x_{t}^{i}, w_{t}^{i}\right)$ is obtained, as the control vector $u_{t}^{i^{F H^{\circ}}}$ depends on the local current state $x_{t}^{i}$ and on the vector of delayed states $w_{t}^{i}$ communicated to the agent $\mathcal{A}^{i}$ by the cooperating agents $\mathcal{G}^{i}=\left\{\mathcal{A}^{j}, j \in G^{i}\right\}$. The system (4) under the action of the $R H$ optimal control law can thus be rewritten as

$$
\begin{array}{r}
x_{t+1}^{i}=\tilde{f}^{i}\left(x_{t}^{i}, w_{t}^{i}\right) \triangleq f^{i}\left(x_{t}^{i}, \gamma_{R H^{\circ}}^{i}\left(x_{t}^{i}, w_{t}^{i}\right)\right), \\
t \geq 0, x_{0}^{i}=\bar{x}^{i}
\end{array}
$$


which is of the same form of the general system (1) considered in Section II. It is worth noting that, from well-known results on RH control (see, for instance, [18] and the references cited therein), we have $\gamma_{R H^{\circ}}^{i}(0,0)=0$ and hence $\tilde{f}^{i}(0,0)=0$, that is, the origin is an equilibrium state for agent $\mathcal{A}^{i}$ when $w_{t}^{i}=0, t \geq 0$.

\section{Stability of the team of COOPERATING AGENTS}

The stability analysis of the team of cooperating agents will be carried out in three main steps. In Subsection IV-A some basic results concerning the regional input-to-state stability properties of general discrete-time systems of the form (1) will be stated and proved, following the approach presented in [17]; the reader is referred to Section II for the definitions regarding the regional ISS property. In Subsection IV-B, the regional stability results will be exploited referring to specific dynamic models (10) of $\mathcal{A}^{i}$. We will indeed prove that each agent is regionally ISS with respect to the input represented by the delayed incoming information from its neighbors. Finally the team of cooperating agents will be considered in Subsection IV-C as a single dynamic system resulting from a feedback interconnection of regionally ISS systems. Showing that both the elements of this interconnection are endowed with ISS-Lyapunov functions, will result in proving the asymptotic stability of the team of cooperating agents by resorting to appropriate small-gain conditions.

\section{A. Regional ISS results}

The regional ISS stability analysis will now be associated to the existence of a suitable Lyapunov function (in general, a-priori non smooth) defined as follows.

Definition 4.1 (ISS-Lyapunov function in $\Xi$ ): A function $V: \mathbb{R}^{n} \times \mathbb{R}^{r} \rightarrow \mathbb{R}_{\geq 0}$ is an ISS-Lyapunov function in $\Xi$ for system (1), if:

1) $\Xi$ is a compact robust positively invariant set including the origin as an interior point;

2) there exist a compact set $\Omega \subseteq \Xi$ (including the origin as an interior point) and suitable $\mathcal{K}_{\infty}$-functions $\alpha_{1}, \alpha_{2}$, $\sigma_{1}$ such that:

$$
\begin{gathered}
V(x, w) \geq \alpha_{1}(|x|), \forall x \in \Xi, \forall w \in W \\
V(x, w) \leq \alpha_{2}(|x|)+\sigma_{1}(|w|), \\
\forall x \in \Omega, \forall w \in W
\end{gathered}
$$

3) there exists a suitable $\mathcal{K}_{\infty}$-functions $\alpha_{3}$ and some $\mathcal{K}$ functions $\sigma_{2}, \sigma_{3}$ such that:

$$
\begin{gathered}
V\left(\tilde{f}\left(x, w_{1}\right), w_{2}\right)-V\left(x, w_{1}\right) \\
\leq-\alpha_{3}(|x|)+\sigma_{2}\left(\left|w_{1}\right|\right)+\sigma_{3}\left(\left|w_{2}\right|\right), \\
\forall x \in \Xi, \forall w_{1}, w_{2} \in W
\end{gathered}
$$

4) there exist some suitable $\mathcal{K}_{\infty}$-functions $\varepsilon$ and $\rho$ ( $\rho$ should be such that $(\operatorname{Id}-\rho)$ is a $\mathcal{K}_{\infty}$-function, too) such that the following compact set $D \subset \Omega$ (including the origin as an interior point) can be defined for some constant $c>0$ :

$$
\begin{aligned}
D \triangleq\{x: d(x, \partial \Omega)>c, V(x, w) & \leq b(\hat{w}), \\
& \forall w \in W\} \subset \Omega,
\end{aligned}
$$

where $b \triangleq \alpha_{4}^{-1} \circ \rho^{-1} \circ \sigma_{4}$, with $\alpha_{4} \triangleq \underline{\alpha}_{3} \circ \bar{\alpha}_{2}^{-1}, \underline{\alpha}_{3}(s) \triangleq$ $\min \left(\alpha_{3}(s / 2), \varepsilon(s / 2)\right), \bar{\alpha}_{2}(s) \triangleq \alpha_{2}(s)+\sigma_{1}(s), \sigma_{4}(s) \triangleq$ $\varepsilon(s)+\sigma_{2}(s)+\sigma_{3}(s)$, and $\hat{w} \triangleq \max _{w}\{|w|: w \in W\}$.

A sufficient condition for regional ISS of system (1) can now be stated.

Theorem 4.1: If the system (1) admits an ISSLyapunov function in $\Xi$, then it is ISS in $\Xi$ and $\lim _{t \rightarrow \infty} d(x(t, \bar{x}, w), D)=0$.

Proof: Let $\bar{x} \in \Xi$. The proof will be carried out in three steps.

Step 1. First, we show that the set $D$ defined in (14) is robust positively invariant for system (1). From the definition of $\bar{\alpha}_{2}(s)$ it follows that $\alpha_{2}(|x|)+\sigma_{1}(|w|) \leq \bar{\alpha}_{2}(|x|+|w|)$. Therefore, $V(x, w) \leq \bar{\alpha}_{2}(|x|+|w|)$ and hence $|x|+|w| \geq$ $\bar{\alpha}_{2}^{-1}(V(x, w))$. Moreover (see [13]):

$$
\alpha_{3}(|x|)+\varepsilon(|w|) \geq \underline{\alpha}_{3}(|x|+|w|) \geq \alpha_{4}(V(x, w))
$$

where $\alpha_{4}(s) \triangleq \underline{\alpha}_{3} \circ \bar{\alpha}_{2}^{-1}(V(x, w))$ is a $\mathcal{K}_{\infty}$-function. Then, let us consider system (1) and the state transition from $x_{t}$ to $x_{t+1}$ :

$$
\begin{aligned}
& V\left(\tilde{f}\left(x_{t}, w_{t}\right), w_{t+1}\right)-V\left(x_{t}, w_{t}\right) \\
& \leq-\alpha_{4}\left(V\left(x_{t}, w_{t}\right)\right)+\varepsilon\left(\left|w_{t}\right|\right) \\
& +\sigma_{2}\left(\left|w_{t}\right|\right)+\sigma_{3}\left(\left|w_{t+1}\right|\right) \\
& \leq-\alpha_{4}\left(V\left(x_{t}, w_{t}\right)\right)+\sigma_{4}(\hat{w}), \\
& \quad \forall x \in \Omega, \forall w_{t}, w_{t+1} \in W, \forall t \geq 0,
\end{aligned}
$$

where $\sigma_{4}(s)=\varepsilon(s)+\sigma_{2}(s)+\sigma_{3}(s)$. Let us now assume that $x_{t_{k}} \in D$. Then $V\left(x_{t_{k}}, w_{t_{k}}\right) \leq b(\hat{w})$; this implies $\rho \circ$ $\alpha_{4}\left(V\left(x_{t_{k}}, w_{t_{k}}\right)\right) \leq \sigma_{4}(\hat{w})$. Without loss of generality, assume that $\left(\operatorname{Id}-\alpha_{4}\right)$ is a $\mathcal{K}_{\infty}$-function, otherwise take a "bigger" $\alpha_{2}$ so that $\underline{\alpha}_{3}<\bar{\alpha}_{2}$. Then

$$
\begin{aligned}
V(\tilde{f} & \left.\left(x_{t_{k}}, w_{t_{k}}\right), w_{t_{k}+1}\right) \\
\leq & \left(\operatorname{Id}-\alpha_{4}\right)\left(V\left(x_{t_{k}}, w_{t_{k}}\right)\right)+\sigma_{4}(\hat{w}) \\
\leq & \left(\operatorname{Id}-\alpha_{4}\right)(b(\hat{w}))+\sigma_{4}(\hat{w}) \\
= & -(\operatorname{Id}-\rho) \circ \alpha_{4}(b(\hat{w}))+b(\hat{w})-\rho \circ \alpha_{4}(b(\hat{w})) \\
& +\sigma_{4}(\hat{w}) .
\end{aligned}
$$

From the definition of $b$, it follows that $\rho \circ \alpha_{4}(b(\hat{w}))=\sigma_{4}(\hat{w})$ and, owing to the fact that $(\mathrm{Id}-\rho)$ is a $\mathcal{K}_{\infty}$-function, we obtain

$$
\begin{aligned}
& V\left(\tilde{f}\left(x_{t_{k}}, w_{t_{k}}\right), w_{t_{k}+1}\right) \\
& \quad \leq-(\operatorname{Id}-\rho) \circ \alpha_{4}(b(\hat{w}))+b(\hat{w}) \leq b(\hat{w}) .
\end{aligned}
$$

By induction one can show that $V\left(\tilde{f}\left(x_{t_{k}+j-1}, w_{t_{k}+j-1}\right), w_{t_{k}+j}\right) \leq b(\hat{w})$ for all $j \in \mathbb{Z}_{\geq 0}$, that is $x_{t} \in D$, for all $t \geq t_{k}$. Hence $D$ is robust positively invariant for system (1).

Step 2. Next, we show that the state, starting from $\Xi \backslash D$, tends asymptotically to $D$. Firstly, if $x \in \Omega \backslash D$, then

$$
\rho \circ \alpha_{4}\left(V\left(x_{t}, w_{t}\right)\right)>\sigma_{4}(\hat{w}) .
$$


From the inequality $\alpha_{3}\left(\left|x_{t}\right|\right)+\varepsilon\left(\left|w_{t}\right|\right) \geq \alpha_{4}\left(V\left(x_{t}, w_{t}\right)\right)$, we have

$$
\rho\left(\alpha_{3}\left(\left|x_{t}\right|\right)+\varepsilon\left(\left|w_{t}\right|\right)\right)>\sigma_{4}(\hat{w}) .
$$

On the other hand, $(\operatorname{Id}-\rho)$ is a $\mathcal{K}_{\infty}$-function, hence

$$
\operatorname{Id}(s)>\rho(s), \forall s>0
$$

then

$$
\begin{aligned}
\alpha_{3}\left(\left|x_{t}\right|\right)+\varepsilon(\hat{w}) & >\alpha_{3}\left(\left|x_{t}\right|\right)+\varepsilon\left(\left|w_{t}\right|\right) \\
& >\rho\left(\alpha_{3}\left(\left|x_{t}\right|\right)+\varepsilon\left(\left|w_{t}\right|\right)\right) \\
& >\sigma_{4}(\hat{w})=\varepsilon(\hat{w})+\sigma_{2}(\hat{w})+\sigma_{3}(\hat{w}) \\
\forall x \in \Omega \backslash D, & \forall w_{t} \in W
\end{aligned}
$$

which, in turn, implies that

$$
\begin{array}{r}
V\left(\tilde{f}\left(x_{t}, w_{t}\right), w_{t+1}\right)-V\left(x_{t}, w_{t}\right) \\
\leq-\alpha_{3}\left(\left|x_{t}\right|\right)+\sigma_{2}(\hat{w})+\sigma_{3}(\hat{w})<0, \\
\forall x_{t} \in \Omega \backslash D, \forall w_{t}, w_{t+1} \in W .
\end{array}
$$

Moreover, in view of (14), $\exists \bar{c}>0$ such that $\forall x_{1} \in \Xi \backslash \Omega$, there exists $x_{2} \in \Omega \backslash D$ such that $\alpha_{3}\left(\left|x_{2}\right|\right) \leq \alpha_{3}\left(\left|x_{1}\right|\right)-\bar{c}$. Then from (16) it follows that

$$
\begin{gathered}
-\alpha_{3}\left(\left|x_{1}\right|\right)+\bar{c} \leq-\alpha_{3}\left(\left|x_{2}\right|\right)<-\sigma_{2}(\hat{w})-\sigma_{3}(\hat{w}), \\
\forall x_{1} \in \Xi \backslash \Omega, \forall x_{2} \in \Omega \backslash D .
\end{gathered}
$$

Then

$$
\begin{aligned}
& V\left(\tilde{f}\left(x_{t}, w_{t}\right), w_{t+1}\right)-V\left(x_{t}, w_{t}\right) \\
& \quad \leq-\alpha_{3}\left(\left|x_{t}\right|\right)+\sigma_{2}(\hat{w})+\sigma_{3}(\hat{w}) \\
& \quad<-\bar{c}, \quad \forall x \in \Xi \backslash \Omega, \forall w_{t}, w_{t+1} \in W,
\end{aligned}
$$

so that there exists $T_{1}$ such that

$$
x\left(T_{1}, \bar{x}, w\right) \in \Omega .
$$

Therefore, starting from $\Xi$, the state will reach the region $\Omega$ in a finite time. If $x_{T_{1}} \in D$, the region $D$ is achieved in a finite time. Since $D$ is robust positively invariant, it is true that $\lim _{t \rightarrow \infty} d(x(t, \bar{x}, w), D)=0$. Otherwise, if $x_{t}=x_{T_{1}} \notin D$, $\rho \circ \alpha_{4}\left(V\left(x_{t}, w_{t}\right)\right)>\sigma_{4}(\hat{w})$; moreover, from (15) we have

$$
\begin{aligned}
V\left(\tilde{f}\left(x_{t}, w_{t}\right), w_{t+1}\right)-V\left(x_{t}, w_{t}\right) \\
\leq-\alpha_{4}\left(V\left(x_{t}, w_{t}\right)\right)+\sigma_{4}(\hat{w}) \\
=-(\operatorname{Id}-\rho) \circ \alpha_{4}\left(V\left(x_{t}, w_{t}\right)\right)-\rho \circ \alpha_{4}\left(V\left(x_{t}, w_{t}\right)\right) \\
\quad+\sigma_{4}(\hat{w}) \\
\leq-(\operatorname{Id}-\rho) \circ \alpha_{4}\left(V\left(x_{t}, w_{t}\right)\right) \\
\leq-(\operatorname{Id}-\rho) \circ \alpha_{4} \circ \alpha_{1}\left(\left|x_{t}\right|\right) \\
\quad \forall x_{t} \in \Omega \backslash D, \forall w_{t}, w_{t+1} \in W,
\end{aligned}
$$

where the last step is obtained using (11). Then, $\forall \varepsilon^{\prime}>$ $0, \exists T_{2}\left(\varepsilon^{\prime}\right) \geq T_{1}$ such that

$$
V\left(x_{T_{2}}, w_{T_{2}}\right) \leq \varepsilon^{\prime}+b(\hat{w}) .
$$

Therefore, starting from $\Xi$, the state will arrive close to $D$ in a finite time and in $D$ asymptotically. Hence $\lim _{k \rightarrow \infty} d(x(k, \bar{x}, w), D)=0$.

Step 3. Finally, we show that system (1) is regionally ISS in $\Xi$. Given $e \in \mathbb{R}_{\geq 0}$, let $R(e) \triangleq\{x: V(x, w) \leq e, \forall w \in W\}$.
Let $\Theta \triangleq\left\{x: V(x, w) \leq \bar{e}=\max _{R(e) \subseteq \Omega} e, \forall w \in W\right\}$. Note that $\bar{e}>b(\hat{w})$ and $D \subset \Theta$. Since the region $D$ is reached asymptotically, the state will arrive in $\Theta$ in a finite time, that is there exists $T_{\theta}$ such that $V\left(x_{k}, w_{k}\right) \leq \bar{e}, \forall k \geq T_{\theta}$. Hence, the region $\Theta$ is a robust positively invariant set for the system (1). Proceeding now as in the Proof of Lemma 3.5 in [30], we can prove that there exist some $\mathcal{K} \mathcal{L}$-function $\hat{\beta}$ and a $\mathcal{K}$ function $\hat{\gamma}$ such that

$$
\begin{gathered}
V\left(x_{t}, w_{t}\right) \leq \max \left\{\hat{\beta}\left(V\left(x_{0}, w_{0}\right), t\right), \hat{\gamma}\left(\| w_{[t]}||\right)\right\}, \\
\forall t \geq 0, \forall x \in \Theta, \forall w \in \mathcal{M}_{W}
\end{gathered}
$$

where $\hat{\gamma}=\alpha_{4}^{-1} \circ \rho^{-1} \circ \sigma_{4}$. Hence

$$
\begin{gathered}
\alpha_{1}\left(\left|x_{t}\right|\right) \leq \max \left\{\hat{\beta}\left(\alpha_{2}\left(\left|x_{0}\right|\right)+\sigma_{1}\left(\left|w_{0}\right|\right), t\right), \hat{\gamma}\left(|| w_{[t]}||\right)\right\}, \\
\leq \max \left\{\hat{\beta}\left(2 \alpha_{2}\left(\left|x_{0}\right|\right), t\right)+\hat{\beta}\left(2 \sigma_{1}\left(\left|w_{0}\right|\right), t\right),\right. \\
\left.\hat{\gamma}\left(|| w_{[t]}||\right)\right\},
\end{gathered}
$$

$\forall t \geq 0, \forall x_{t} \in \Theta, \forall w \in \mathcal{M}_{W}$

The last step is obtained considering that $\hat{\beta}(r+s, t) \leq$ $\hat{\beta}(2 r, t)+\hat{\beta}(2 s, t)$ (see [13]). Then

$$
\begin{aligned}
&\left|x_{t}\right| \leq \max \left\{\hat{\beta}_{1}\left(\alpha_{2}\left(\left|x_{0}\right|\right), t\right)+\hat{\beta}_{1}\left(\sigma_{1}\left(\left|w_{0}\right|\right), t\right),\right. \\
&\left.\hat{\gamma}_{1}\left(|| w_{[t]} \mid\right)\right\}, \\
& \leq \hat{\beta}_{1}\left(\alpha_{2}\left(\left|x_{0}\right|\right), t\right)+\hat{\beta}_{1}\left(\sigma_{1}\left(\left|w_{0}\right|\right), t\right) \\
&+\hat{\gamma}_{1}\left(|| w_{[t]}||\right) \\
& \leq \hat{\beta}_{1}\left(\alpha_{2}\left(\left|x_{0}\right|\right), t\right)+\hat{\beta}_{1}\left(\sigma_{1}\left(|| w_{[t]}||\right), 0\right) \\
&+\hat{\gamma}_{1}\left(|| w_{[t]}||\right) \\
& \leq \hat{\beta}_{2}\left(\left|x_{0}\right|, t\right)+\hat{\gamma}_{2}\left(|| w_{[t]}||\right), \\
& \forall t \geq 0, \forall x_{t} \in \Theta, \quad \forall w \in \mathcal{M}_{W}
\end{aligned}
$$

where $\hat{\beta}_{1}(s, t) \triangleq\left(\alpha_{1}^{-1}\right) \circ \beta_{1}(2 s, t)$ and $\hat{\beta}_{2}(s, t) \triangleq \hat{\beta}_{1}\left(\alpha_{2}(s), t\right)$ are $\mathcal{K} \mathcal{L}$-functions while $\hat{\gamma}_{1}(s) \triangleq\left(\alpha_{1}^{-1}\right) \circ \hat{\gamma}(s)$ and $\hat{\gamma}_{2}(s) \triangleq$ $\hat{\beta}_{1}\left(\sigma_{1}\left(\left\|w_{[t]}\right\|\right), 0\right)+\hat{\gamma}_{1}\left(\left\|w_{[t]}\right\|\right)$ are $\mathcal{K}$-functions. So, by (18) the system (1) is $I S S$ in $\Theta$. The regional $I S S$ property in $\Theta$ is equivalent to $U A G$ in $\Theta$ and $L S$ (see [32] for the continuous time and [33] for the discrete time case). Using the fact that $\Theta$ is achieved in a finite time, $U A G$ in $\Theta$ implies $U A G$ in $\Xi$. Then $U A G$ in $\Xi$ and $L S$ imply $I S S$ in $\Xi$.

To sum up, in this subsection an important sufficient condition for regional ISS of constrained systems of the form (1) has been stated and proved. In the next subsection, Theorem 4.1 will be exploited with reference to each agent $\mathcal{A}^{i}$ under the action of the local RH control law.

\section{B. Stability properties of the single agents}

Let us consider a generic agent $\mathcal{A}^{i}$ whose dynamics is described by (4). By exploiting the results proved in Subsection IV-A, we will now show that each agent $\mathcal{A}^{i}$, with $i=1,2, \ldots, M$ is regionally ISS with respect to the inputs represented by the information vectors $w_{t}^{i}$ received from its cooperating agents at each time-step $t$. Clearly, in this context, we are considering each agent as a "separate" dynamic system in the team, in the sense that the input vectors $w_{t}^{i}$ are "external" variables that are assumed not to depend on the 
behavior of the other cooperating agents (i.e., at the present stage, the coupling between the agents is not directly taken into account). Let now introduce some further useful assumptions and definitions.

Assumption 1: A terminal cost function $h_{f}^{i}$, a final constraint set $X_{f}^{i}$, and an auxiliary control law $\kappa_{f}^{i}$ are given such that:

1) $X_{f}^{i} \subset X^{i}, X_{f}^{i}$ closed, $0 \in X_{f}^{i}$;

2) $\kappa_{f}^{i}\left(x^{i}\right) \in U^{i},\left|\kappa_{f}^{i}\left(x^{i}\right)\right| \leq L_{\kappa_{f}}^{i}\left|x^{i}\right|, L_{\kappa_{f}}^{i}>0, \forall x^{i} \in X_{f}^{i}$;

3) $\left|f^{i}\left(x^{i}, \kappa_{f}^{i}\left(x^{i}\right)\right)\right| \leq L_{f_{c}}^{i}\left|x^{i}\right|, L_{f_{c}}^{i}>0, \forall x^{i} \in X_{f}^{i}$;

4) $f^{i}\left(x^{i}, \kappa_{f}^{i}\left(x^{i}\right)\right) \in X_{f}^{i}, \forall x^{i} \in X_{f}^{i}$;

5) $\alpha_{h_{f}^{i}}\left(\left|x^{i}\right|\right) \leq h_{f}^{i}\left(x^{i}\right) \leq \beta_{h_{f}^{i}}\left(\left|x^{i}\right|\right), \forall x^{i} \in X_{f}^{i}$, where $\alpha_{h_{f}^{i}}$ and $\beta_{h_{f}^{i}}$ are $\mathcal{K}_{\infty}$-functions;

6) $h_{f}^{i}\left(f^{i}\left(x^{i}, \kappa_{f}^{i}\left(x^{i}\right)\right)\right)-h_{f}^{i}\left(x^{i}\right) \leq-h^{i}\left(x^{i}, \kappa_{f}^{i}\left(x^{i}\right)\right)-$ $q^{i}\left(x^{i}, \tilde{w}^{i}\right)+\psi^{i}\left(\left|\tilde{w}^{i}\right|\right), \forall x^{i} \in X_{f}^{i}, \forall w^{i} \in W^{i}$, where $\psi^{i}$ is a $\mathcal{K}$-function and $\tilde{w}^{i} \triangleq\left(A_{w}^{i}\right)^{N_{p}^{i}-1} w^{i}$.

Assumption 2: The partial cost function $h^{i}$ is such that $\underline{r}^{i}\left(\left|x^{i}\right|\right) \leq h^{i}\left(x^{i}, u^{i}\right), \forall x^{i} \in X^{i}, \forall u^{i} \in U^{i}$ where $\underline{r}^{i}$ is a $\mathcal{K}_{\infty}$-function. Moreover, $h^{i}$ is Lipschitz with respect to $x^{i}$ and $u^{i}$ in $X^{i} \times U^{i}$, with Lipschitz constants denoted as $L_{h}^{i}$ and $L_{h u}^{i}$, respectively.

Assumption 3: The cooperation cost function $q^{i}$ is such that $0 \leq q^{i}\left(x^{i}, w^{i}\right), \forall x^{i} \in X^{i}, \forall w^{i} \in W^{i}$. Moreover $q^{i}$ is Lipschitz with respect to $x^{i}$ and $w^{i}$ in $X^{i} \times W^{i}$, with Lipschitz constants denoted as $L_{q}^{i}$ and $L_{q w}^{i}$, respectively.

Assumption 4: Let $X^{i^{\kappa_{f}}}$ denote the set of states $x_{t}^{i}$ of the system (4) for which $\tilde{u}_{t, t+N_{c}^{i}-1} \triangleq$ $\operatorname{col}\left[\kappa_{f}^{i}\left(x_{t}^{i}\right), \kappa_{f}^{i}\left(x_{t+1}^{i}\right), \ldots, \kappa_{f}^{i}\left(x_{t+N_{c}^{i}-1}^{i}\right)\right]$ is an admissible control sequence for the FH optimal control Problem 3.1 and for which Points 2 and 3 of Assumption 1 are satisfied. Moreover, suppose ${ }^{2}$ that $L_{f_{c}}^{i} \neq 1$ and let $V^{i}\left(x_{t}^{i}, w_{t}^{i}\right) \triangleq J_{F H}^{i}\left(x_{t}^{i}, w_{t}^{i}, u_{t, t+N_{c}^{i}-1}^{i^{F H^{\circ}}}, N_{c}^{i}, N_{p}^{i}\right), \Omega=X^{i^{\kappa_{f}}}$, $\alpha_{1}=\underline{r}^{i}, \alpha_{2}\left(\left|x_{t}^{i}\right|\right)=\left(L_{h}^{i}+L_{h u}^{i} L_{\kappa_{f}}^{i}+L_{q}^{i}\right) \frac{\left(L_{f_{c}}^{i}\right)^{N_{p}^{i}}-1}{L_{f_{c}}^{i}-1}\left|x_{t}^{i}\right|+$ $\beta_{h_{f}^{i}}\left(\left(L_{f_{c}}^{i}\right)^{N_{p}^{i}}\left|x_{t}^{i}\right|\right), \alpha_{3}=\underline{r}^{i}, \quad \sigma_{1}=L_{q w}^{i} \frac{\left(\alpha_{w}^{i}\right)^{N_{p}^{i}}-1}{\alpha_{w}^{i}-1}$, $\sigma_{2}\left(\left|w_{t}^{i}\right|\right)=\alpha_{w}^{i} L_{q w}^{i} \frac{\left(\alpha_{w}^{i}\right)^{N_{p}^{i}}-1}{\alpha_{w}^{i}-1}\left|w_{t}^{i}\right|+\psi^{i}\left(\left(\alpha_{w}^{i}\right)^{N_{p}^{i}-1}\left|w_{t}^{i}\right|\right)$, and $\sigma_{3}=L_{q w}^{i} \frac{\left(\alpha_{w}^{i}\right)^{N_{p}^{i}}-1}{\alpha_{w}^{i}-1}$. The set $W^{i}$ is such that (14) is satisfied.

The main result can now be stated.

Theorem 4.2: Under Assumptions 1-4, the locallycontrolled agent $\mathcal{A}^{i}, i=1, \ldots, M$, whose closed-loop dynamics are described by (10), subject to constraints (5), (6), and (7), is ISS with robust output admissible set $X^{i^{M P C}}$,

\footnotetext{
${ }^{2}$ The very special case $L_{f_{c}}^{i}=1$ can be trivially addressed by a few suitable modifications to the proof of Theorem 4.2.
}

where $X^{i^{M P C}}$ denotes the set of states of system (4) for which a solution of the FH optimal control Problem 3.1 does exist. sequence

Proof: First, by Assumption 1, for any $x_{t}^{i} \in X_{f}^{i}$, the

$$
\tilde{u}_{t, t+N_{c}^{i}-1}^{i}=\operatorname{col}\left[\kappa_{f}^{i}\left(x_{t}^{i}\right), \kappa_{f}^{i}\left(x_{t+1}^{i}\right), \ldots, \kappa_{f}^{i}\left(x_{t+N_{c}^{i}-1}^{i}\right)\right]
$$

is an admissible control sequence for the $\mathrm{FH}$ optimal control Problem 3.1 (see Definition 3.1). Then $X^{i^{M P C}} \supseteq X^{i^{\kappa_{f}}} \supseteq$ $X_{f}^{i}$. By Theorem 4.1, if system admits an ISS-Lyapunov function in $X^{i^{M P C}}$, then it is ISS in $X^{i^{M P C}}$. In this respect, in the following it will be shown that $V^{i}\left(x_{t}^{i}, w_{t}^{i}\right) \triangleq$ $J_{F H}^{i}\left(x_{t}^{i}, w_{t}^{i}, u_{t, t+N_{c}^{i}-1}^{i^{\circ}{ }^{\circ}}, N_{c}^{i}, N_{p}^{i}\right)$ is an ISS-Lyapunov function in $X^{i^{M P C}}$. Moreover, in view of Point 5 of Assumption 1 and Assumptions 2-3

$$
\begin{aligned}
V^{i}\left(x_{t}^{i}, w_{t}^{i}\right) \leq & J_{F H}^{i}\left(x_{t}^{i}, w_{t}^{i}, \tilde{u}_{t, t+N_{c}^{i}-1}^{i}, N_{c}^{i}, N_{p}^{i}\right) \\
\leq & \sum_{l=t}^{t+N_{p}^{i}-1}\left\{L_{h}^{i}\left|x_{l}^{i}\right|+L_{h u}^{i}\left|\kappa_{f}\left(x_{l}^{i}\right)\right|\right. \\
& \left.+L_{q}^{i}\left|x_{l}^{i}\right|+L_{q w}^{i}\left|w_{l}^{i}\right|\right\}+\beta_{h_{f}^{i}}\left(\left|x_{t+N_{p}^{i}}^{i}\right|\right) \\
\leq & \sum_{l=t}^{t+N_{p}^{i}-1}\left\{\left(L_{h}^{i}+L_{h u}^{i} L_{\kappa_{f}}^{i}+L_{q}^{i}\right)\left|x_{l}^{i}\right|\right. \\
& \left.+L_{q w}^{i}\left|w_{l}^{i}\right|\right\},+\beta_{h_{f}^{i}}\left(\left|x_{t+N_{p}^{i}}^{i}\right|\right)
\end{aligned}
$$

so that in view of Point 3 of Assumption 1 and owing to (8), we have

$$
\begin{aligned}
V^{i}\left(x_{t}^{i}, w_{t}^{i}\right) \leq & \sum_{l=t}^{t+N_{p}^{i}-1}\left\{\left(L_{h}^{i}+L_{h u}^{i} L_{\kappa_{f}}^{i}+L_{q}^{i}\right)\left(L_{f_{c}}^{i}\right)^{l-t}\left|x_{t}^{i}\right|\right. \\
& \left.+L_{q w}^{i}\left(\alpha_{w}^{i}\right)^{l-t}\left|w_{t}^{i}\right|\right\}+\beta_{h_{f}^{i}}\left(\left(L_{f_{c}}^{i}\right)^{N_{p}^{i}}\left|x_{t}^{i}\right|\right) \\
\leq & \left(L_{h}^{i}+L_{h u}^{i} L_{\kappa_{f}}^{i}+L_{q}^{i}\right) \frac{\left(L_{f_{c}}^{i}\right)^{N_{p}^{i}}-1}{L_{f_{c}}^{i}-1}\left|x_{t}^{i}\right| \\
& +L_{q w}^{i} \frac{\left(\alpha_{w}^{i}\right)^{N_{p}^{i}}-1}{\alpha_{w}^{i}-1}\left|w_{t}^{i}\right|+\beta_{h_{f}^{i}}\left(\left(L_{f_{c}}^{i}\right)^{N_{p}^{i}}\left|x_{t}^{i}\right|\right) .
\end{aligned}
$$

Hence there exist two $\mathcal{K}_{\infty}$-functions $\beta^{i}$ and $\sigma^{i}$ such that the following upper bound is verified:

$$
\begin{gathered}
V^{i}\left(x_{t}^{i}, w_{t}^{i}\right) \leq \beta^{i}\left(\left|x_{t}^{i}\right|\right)+\sigma^{i}\left(\left|w_{t}^{i}\right|\right), \\
\forall x_{t}^{i} \in X^{i^{\kappa} f_{f}}, \forall w_{t}^{i} \in W^{i} .
\end{gathered}
$$

The lower bound on $V^{i}\left(x_{t}^{i}, w_{t}^{i}\right)$ is easily obtained using Assumption 2:

$$
V^{i}\left(x_{t}^{i}, w_{t}^{i}\right) \geq \underline{r}^{i}\left(\left|x_{t}^{i}\right|\right), \forall x_{t}^{i} \in X^{i}, \forall w_{t}^{i} \in W^{i}
$$

Now, in view of Assumption 1, it turns out that

$$
\bar{u}_{t+1, t+N_{c}^{i}}^{i} \triangleq \operatorname{col}\left(u_{t+1, t+N_{c}^{i}-1}^{i^{F H^{\circ}}}, \kappa_{f}^{i}\left(x_{t+N_{c}^{i}}^{i}\right)\right)
$$


is an admissible (in general, suboptimal) control sequence for the FH optimal control Problem 3.1 at time $t+1$ with cost

$$
\begin{aligned}
J_{F H}^{i}( & \left.x_{t+1}^{i}, w_{t+1}^{i}, \bar{u}_{t+1, t+N_{c}^{i}}, N_{c}^{i}, N_{p}^{i}\right) \\
= & V^{i}\left(x_{t}^{i}, w_{t}^{i}\right)-h^{i}\left(x_{t}^{i}, u_{t, t}^{i^{F H^{\circ}}}\right)-q^{i}\left(x_{t}^{i}, w_{t}^{i}\right) \\
& +\sum_{l=t+1}^{t+N_{p}^{i}-1}\left\{h^{i}\left(x_{l}^{i}, \bar{u}_{l}^{i}\right)+q^{i}\left(x_{l}^{i},\left(A_{w}^{i}\right)^{l-(t+1)} w_{t+1}^{i}\right)\right. \\
& \left.-h^{i}\left(x_{l}^{i}, u_{l}^{i^{F H^{\circ}}}\right)-q^{i}\left(x_{l}^{i},\left(A_{w}^{i}\right)^{l-t} w_{t}^{i}\right)\right\} \\
& +h^{i}\left(x_{t+N_{p}^{i}}^{i}, \kappa_{f}^{i}\left(x_{t+N_{p}^{i}}^{i}\right)\right) \\
& +q^{i}\left(x_{t+N_{p}^{i}}^{i},\left(A_{w}^{i}\right)^{N_{p}^{i}-1} w_{t+1}^{i}\right) \\
& +h_{f}^{i}\left(f^{i}\left(x_{t+N_{p}^{i}}^{i}, \kappa_{f}^{i}\left(x_{t+N_{p}^{i}}^{i}\right)\right)\right)-h_{f}^{i}\left(x_{t+N_{p}^{i}}^{i}\right)
\end{aligned}
$$

Noting that, using Assumption 3

$$
\begin{aligned}
q^{i} & \left(x_{l}^{i},\left(A_{w}^{i}\right)^{l-(t+1)} w_{t+1}^{i}\right)-q^{i}\left(x_{l}^{i},\left(A_{w}^{i}\right)^{l-t} w_{t}^{i}\right) \\
& \leq\left|q^{i}\left(x_{l}^{i},\left(A_{w}^{i}\right)^{l-(t+1)} w_{t+1}^{i}\right)-q^{i}\left(x_{l}^{i},\left(A_{w}^{i}\right)^{l-t} w_{t}^{i}\right)\right| \\
& \leq L_{q w}^{i}\left|\left(A_{w}^{i}\right)^{l-(t+1)} w_{t+1}^{i}-\left(A_{w}^{i}\right)^{l-t} w_{t}^{i}\right| \\
& =L_{q w}^{i}\left(\alpha_{w}^{i}\right)^{l-(t+1)}\left|w_{t+1}^{i}-A_{w}^{i} w_{t}^{i}\right| \\
& \leq L_{q w}^{i}\left(\alpha_{w}^{i}\right)^{l-(t+1)}\left(\left|w_{t+1}^{i}\right|+\alpha_{w}^{i}\left|w_{t}^{i}\right|\right),
\end{aligned}
$$

and by using Point 6 of Assumption 1, we obtain

$$
\begin{aligned}
& J_{F H}^{i}\left(x_{t+1}^{i}, w_{t+1}^{i}, \bar{u}_{t+1, t+N_{c}^{i}}, N_{c}^{i}, N_{p}^{i}\right) \\
& \leq V^{i}\left(x_{t}^{i}, w_{t}^{i}\right)-h^{i}\left(x_{t}^{i}, u_{t, t}^{i^{F H^{\circ}}}\right)-q^{i}\left(x_{t}^{i}, w_{t}^{i}\right) \\
& +\sum_{l=t+1}^{t+N_{p}^{i}-1} L_{q w}^{i}\left(\alpha_{w}^{i}\right)^{l-(t+1)}\left(\left|w_{t+1}^{i}\right|+\alpha_{w}^{i}\left|w_{t}^{i}\right|\right) \\
& +\psi^{i}\left(\left|\left(A_{w}^{i}\right)^{N_{p}^{i}-1} w_{t}^{i}\right|\right) \\
& \leq V^{i}\left(x_{t}^{i}, w_{t}^{i}\right)-h^{i}\left(x_{t}^{i}, u_{t, t}^{i^{F H^{\circ}}}\right)-q^{i}\left(x_{t}^{i}, w_{t}^{i}\right) \\
& +\varphi_{1}^{i}\left(\left|w_{t}^{i}\right|\right)+\varphi_{2}^{i}\left(\left|w_{t+1}^{i}\right|\right)
\end{aligned}
$$

where

$$
\begin{aligned}
\varphi_{1}^{i}\left(\left|w_{t}^{i}\right|\right) \triangleq & \alpha_{w}^{i} L_{q w}^{i} \frac{\left(\alpha_{w}^{i}\right)^{N_{p}^{i}}-1}{\alpha_{w}^{i}-1}\left|w_{t}^{i}\right| \\
& +\psi^{i}\left(\left(\alpha_{w}^{i}\right)^{N_{p}^{i}-1}\left|w_{t}^{i}\right|\right) \\
\varphi_{2}^{i} \triangleq & L_{q w}^{i} \frac{\left(\alpha_{w}^{i}\right)^{N_{p}^{i}}-1}{\alpha_{w}^{i}-1}
\end{aligned}
$$

are $\mathcal{K}_{\infty}$-functions.

Now, from inequality

$$
V^{i}\left(x_{t+1}^{i}, w_{t+1}^{i}\right) \leq J_{F H}^{i}\left(x_{t+1}^{i}, w_{t+1}^{i}, \bar{u}_{t+1, t+N_{c}^{i}}, N_{c}^{i}, N_{p}^{i}\right)
$$

it follows that

$$
\begin{gathered}
V^{i}\left(x_{t+1}^{i}, w_{t+1}^{i}\right)-V^{i}\left(x_{t}^{i}, w_{t}^{i}\right) \\
\leq-\underline{r}^{i}\left(\left|x_{t}\right|\right)+\varphi_{1}^{i}\left(\left|w_{t}^{i}\right|\right)+\varphi_{2}^{i}\left(\left|w_{t+1}^{i}\right|\right) \\
\forall x_{t}^{i} \in X^{i}, \forall w^{i} \in W^{i} .
\end{gathered}
$$

Finally, in view of the admissible control sequence (21), it follows that $X^{i^{M P C}}$ is a robust positively invariant set for the closed loop (10). Therefore, by (20), (19), (22) and Assumption 4 , the optimal cost $J_{F H}^{i}\left(x_{t}^{i}, w_{t}^{i}, u_{t, t+N_{c}^{i}-1}^{i^{F H^{\circ}}}, N_{c}^{i}, N_{p}^{i}\right)$ is an
$I S S$-Lyapunov function for the closed-loop system (10) in $X^{i^{M P C}}$ and hence the closed-loop system is $I S S$ in $X^{i^{M P C}}$.

It is worth noting that, from the perspective of determining regionally ISS stabilizing control laws, a key aspect is the design of an auxiliary control law $\kappa_{f}^{i}\left(x^{i}\right)$ such that Assumption 1 holds. In this respect, under slightly more restrictive hypotheses on the agents' dynamic models and on the $\mathrm{FH}$ cost function, we give the following useful result (the proof is reported in the Appendix).

Lemma 4.1: Assume that $f^{i} \in \mathscr{C}^{2}, h^{i}\left(x^{i}, u^{i}\right)=$ $x^{i^{\top}} Q^{i} x^{i}+u^{i^{\top}} R^{i} u^{i}, q^{i}\left(x^{i}, w^{i}\right) \leq x^{i^{\top}} \tilde{S}^{i} x^{i^{\top}}+\psi^{i}\left(\left|w^{i}\right|\right)$ with $Q^{i}, R^{i}$, and $\tilde{S}^{i}$ being positive definite matrices and $\psi^{i}$ being a $\mathcal{K}$-function. Furthermore, suppose that there exists a matrix $K^{i}$ such that $A_{c l}^{i}=A^{i}+B^{i} K^{i}$ is stable with $\left.A^{i} \triangleq \frac{\partial f^{i}}{\partial x^{i}}\right|_{x^{i}=0 ; u^{i}=0},\left.B^{i} \triangleq \frac{\partial f^{i}}{\partial u^{i}}\right|_{x^{i}=0 ; u^{i}=0}$. Let $\tilde{Q}^{i} \triangleq$ $\beta^{i}\left(Q^{i}+K^{i^{\top}} R^{i} K^{i}+\tilde{S}_{i}\right)$ with $\beta^{i}>1$, and denote by $\Pi^{i}$ the unique symmetric positive definite solution of the following Lyapunov equation:

$$
A_{c l}^{i^{\top}} \Pi^{i} A_{c l}^{i}-\Pi^{i}+\tilde{Q}^{i}=0 .
$$

Then, there exist a constant $\Upsilon^{i} \in \mathbb{R}_{\geq 0}, \Upsilon^{i}>0$ and a finite integer $\bar{N}_{p}$ such that for all $N_{p} \geq \bar{N}_{p}$ the final set $X_{f}^{i} \triangleq\left\{x^{i} \in \mathbb{R}^{n^{i}}: x^{i^{\top}} \Pi^{i} x^{i} \leq \Upsilon^{i}\right\}$ satisfies Assumption 1 with $\kappa_{f}^{i}\left(x^{i}\right)=K^{i} x^{i}, h_{f}^{i}=x^{i^{\top}} \Pi^{i} x^{i}$.

In the next subsection, the stability analysis of the whole team of agents will be addressed.

\section{Stability properties of the team of agents}

In this subsection, the coupling effects due to the exchange of the delayed state information between the cooperating agents will be taken into account in the context of the stability analysis of the whole team of agents. In this respect, let us consider the team $\mathcal{A}=\left\{\mathcal{A}^{i}, i=1, \ldots, M\right\}$ where each cooperating agent $\mathcal{A}^{i}$ is controlled by the regionally ISSstabilizing RH control local law solving Problem 3.1 for each $i=1, \ldots, M$. Therefore, we can write

$$
\begin{aligned}
x_{t+1}^{1} & =\tilde{f}^{1}\left(x_{t}^{1}, w_{t}^{1}\right) \triangleq f^{1}\left(x_{t}^{1}, \gamma^{1}\left(x_{t}^{1}, w_{t}^{1}\right)\right), \\
x_{t+1}^{2} & =\tilde{f}^{2}\left(x_{t}^{2}, w_{t}^{2}\right) \triangleq f^{2}\left(x_{t}^{2}, \gamma^{2}\left(x_{t}^{2}, w_{t}^{2}\right)\right), \\
& \vdots \\
x_{t+1}^{M} & =\tilde{f}^{M}\left(x_{t}^{M}, w_{t}^{M}\right) \triangleq f^{M}\left(x_{t}^{M}, \gamma^{M}\left(x_{t}^{M}, w_{t}^{M}\right)\right),
\end{aligned}
$$

where, for the sake of notational simplicity, we drop the subscript " $R H^{\mathrm{o}}$ " thus denoting by $\gamma^{i}$ the $\mathrm{RH}$ control law $\gamma_{R H^{\circ}}^{i}$

First of all, let us rewrite the team of dynamical systems as a suitable interconnection of two composite systems. To this end, let

$$
\mathcal{X}_{t} \triangleq \operatorname{col}\left(x_{t}^{1}, \cdots, x_{t}^{M}\right), \overline{\mathcal{W}}_{t} \triangleq \operatorname{col}\left(w_{t}^{1}, \cdots, w_{t}^{M}\right) .
$$


Hence the following state equation can be written:

$$
\mathcal{X}_{t+1}=\tilde{F}\left(\mathcal{X}_{t}, \overline{\mathcal{W}}_{t}\right)
$$

where

$$
\tilde{F}\left(\mathcal{X}_{t}, \overline{\mathcal{W}}_{t}\right) \triangleq \operatorname{col}\left[\tilde{f}^{1}\left(x_{t}^{1}, w_{t}^{1}\right), \cdots, \tilde{f}^{M}\left(x_{t}^{M}, w_{t}^{M}\right)\right] .
$$

Vector $\overline{\mathcal{W}}_{t}$ can be easily characterized as the output of a system describing the delay dynamics of the information exchange process among the agents. For the sake of simplicity and without loss of generality, we assume that $\operatorname{dim}\left(w_{t}^{i}\right) \geq$ $1, i=1, \ldots, M$, that is, we assume that each agent receives at least one delayed state information from another neighboring agent. First, we set $\Delta \triangleq \max \left\{\Delta_{i j}, i, j=\right.$ $1, \ldots, M, i \neq j\}$. Then, we introduce the state vector $\mathcal{Z}_{t} \triangleq \operatorname{col}\left(\rho_{t}^{1}, \cdots, \rho_{t}^{\tau}, \cdots, \rho_{t}^{\Delta}\right), \mathcal{Z}_{t} \in \mathbb{R}^{n_{\mathcal{Z}}}$, where $n_{\mathcal{Z}} \triangleq$ $\operatorname{dim}\left(\mathcal{Z}_{t}\right)$ and where the variables $\rho$ are introduced to store the delayed states; specifically $\rho_{t+1}^{1}=\mathcal{X}_{t}$ and $\rho_{t+1}^{\tau}=\rho_{t}^{\tau-1}, \tau=$ $2, \ldots, \Delta$. Hence, it follows that

$$
\left\{\begin{array}{l}
\mathcal{Z}_{t+1}=A \mathcal{Z}_{t}+B \mathcal{X}_{t} \\
\overline{\mathcal{W}}_{t}=C \mathcal{Z}_{t}
\end{array}\right.
$$

where

$$
\begin{aligned}
& A=\left[\begin{array}{ccccc}
\emptyset & \cdots & \cdots & \cdots & \emptyset \\
I^{1} & \emptyset & \cdots & \cdots & \emptyset \\
\emptyset & I^{2} & \emptyset & \cdots & \emptyset \\
\vdots & \vdots & \vdots & \ddots & \vdots \\
\emptyset & \cdots & \cdots & I^{\Delta-1} & \emptyset
\end{array}\right] \\
& B=\left[\begin{array}{c}
I^{0} \\
\emptyset \\
\vdots \\
\emptyset
\end{array}\right], C=\left[\begin{array}{c}
C^{1} \\
C^{2} \\
\vdots \\
C^{M}
\end{array}\right] \\
& C^{i}=\left[\begin{array}{lllll}
C^{i}(1) & \cdots & C^{i}(\tau) & \cdots & C^{i}(\Delta)
\end{array}\right], \\
& C^{i}(\tau)=\left[\begin{array}{ccccc}
\delta^{i 1}(\tau) & \emptyset & \cdots & \cdots & \emptyset \\
\emptyset & \delta^{i 2}(\tau) & \emptyset & \cdots & \emptyset \\
\emptyset & \cdots & \delta^{i 3}(\tau) & \cdots & \emptyset \\
\vdots & \vdots & \vdots & \ddots & \vdots \\
\emptyset & \cdots & \cdots & \emptyset & \delta^{i M}(\tau)
\end{array}\right] .
\end{aligned}
$$

All matrices $I^{\tau}$, for $\tau=0, \ldots, \Delta-1$ are identity matrices of dimension $n^{\text {tot }} \times n^{\text {tot }}$, where $n^{\text {tot }} \triangleq \operatorname{dim}\left(\mathcal{X}_{t}\right)$ and $\delta^{i j}(\tau) \triangleq I$ is equal to the identity matrix of dimension $n^{j}$, $i, j=1, \ldots, M, i \neq j$ only for $\tau$ corresponding to the delay associated with the information received by the $i$-th from the $j$-th agent; otherwise $\delta^{i j}(\tau)=0$, thus there is no replication of information. It is worth noting that agent $\mathcal{A}^{i}$ does not get replicated information from agent $\mathcal{A}^{j}$, thus in matrix $C$ the matrix $\delta^{i j}(\tau)$ is equal to the identity for only one value of $\tau$.

Summing up, the overall state equation describing the dynamics of the team of agents can be written as a feedback interconnection between the systems described by the state equations (24) and (25). We will now show that an ISSLyapunov function can be defined for each of these systems, which implies that both will turn out to be regionally ISS. After this step, the stability properties of the team of agents will be analyzed by resorting to nonlinear small-gain theorem arguments. First, we let $\mathscr{W} \triangleq W^{1} \times \cdots \times W^{M}, \mathscr{X} \triangleq$ $X^{1} \times \cdots \times X^{M}, \mathscr{X}_{f} \triangleq X_{f}^{1} \times \cdots \times X_{f}^{M}, \mathscr{X}^{M P C} \triangleq$ $X^{1^{M P C}} \times \cdots \times X^{M^{M P C}}, D \triangleq D^{1} \times \cdots \times D^{M}$ and $\hat{\mathcal{W}} \triangleq$ $\max _{\overline{\mathcal{W}}}\{|\overline{\mathcal{W}}|: \overline{\mathcal{W}} \in \mathscr{W}\}$. The following intermediate result can now be proved.

Lemma 4.2: Under Assumptions 1-4, dynamic systems (24) and (25) are provided with suitable ISS-Lyapunov functions $V\left(\mathcal{X}_{t}, \overline{\mathcal{W}}_{t}\right)$ in $\mathscr{X}^{M P C}$ and $V^{D}\left(\mathcal{Z}_{t}\right)$ in $\mathbb{R}^{n_{\mathcal{Z}}}$, respectively.

Proof: Let us consider the ISS-Lyapunov function candidate

$$
V\left(\mathcal{X}_{t}, \overline{\mathcal{W}}_{t}\right) \triangleq \sum_{i=1}^{M} V^{i}\left(x_{t}^{i}, w_{t}^{i}\right)
$$

for system (24). ${ }^{3}$ From (20) and (19), it follows that

$$
\sum_{i=1}^{M} \underline{r}^{i}\left(\left|x_{t}^{i}\right|\right) \leq V\left(\mathcal{X}_{t}, \overline{\mathcal{W}}_{t}\right) \leq \sum_{i=1}^{M} \beta^{i}\left(\left|x_{t}^{i}\right|\right)+\sum_{i=1}^{M} \sigma^{i}\left(\left|w_{t}^{i}\right|\right)
$$

Clearly $\left|x_{t}^{i}\right| \leq\left|\mathcal{X}_{t}\right|$ and $\left|w_{t}^{i}\right| \leq\left|\overline{\mathcal{W}}_{t}\right|, \forall i=1, \ldots, M$ and thus

$$
\begin{aligned}
V\left(\mathcal{X}_{t}, \overline{\mathcal{W}}_{t}\right) & \leq \sum_{i=1}^{M} \beta^{i}\left(\left|x_{t}^{i}\right|\right)+\sum_{i=1}^{M} \sigma^{i}\left(\left|w_{t}^{i}\right|\right) \\
& \leq \sum_{i=1}^{M} \beta^{i}\left(\left|\mathcal{X}_{t}\right|\right)+\sum_{i=1}^{M} \sigma^{i}\left(\left|\overline{\mathcal{W}}_{t}\right|\right) \\
& \leq \beta\left(\left|\mathcal{X}_{t}\right|\right)+\sigma\left(\left|\overline{\mathcal{W}}_{t}\right|\right),
\end{aligned}
$$

where $\quad$ we $\quad$ set $\quad \beta\left(\left|\mathcal{X}_{t}\right|\right) \triangleq \sum_{i=1}^{M} \beta^{i}\left(\left|\mathcal{X}_{t}\right|\right) \quad$ and $\sigma\left(\left|\overline{\mathcal{W}}_{t}\right|\right) \triangleq \sum_{i=1}^{M} \sigma^{i}\left(\left|\overline{\mathcal{W}}_{t}\right|\right)$

Moreover

$$
\sum_{i=1}^{M}\left|x_{t}^{i}\right| \leq \sum_{i=1}^{M}\left|\mathcal{X}_{t}\right|=M\left|\mathcal{X}_{t}\right|
$$

Then $\left|\mathcal{X}_{t}\right| \geq \frac{1}{M} \sum_{i=1}^{M}\left|x_{t}^{i}\right|$ and $\left|\mathcal{X}_{t}\right| \leq \sum_{i=1}^{M}\left|x_{t}^{i}\right|$. Now, recall that, for any $\mathcal{K}$ function $\gamma$, we have $\gamma\left(\sum_{i=1}^{M} a_{i}\right) \leq \sum_{i=1}^{M} \gamma\left(M a_{i}\right)$ where $a_{i}>0, i=1, \ldots, M$ are arbitrarily chosen positive scalars). Therefore, considering the $\mathcal{K}$ function $\underline{r}^{i}$, for a generic $i \in\{1, \ldots, M\}$, we have $\underline{r}^{i}\left(\left|\mathcal{X}_{t}\right|\right) \leq$ $\underline{r}^{i}\left(\sum_{i=1}^{M}\left|x_{t}^{i}\right|\right) \leq \sum_{i=1}^{M} \underline{r}^{i}\left(M\left|x_{t}^{i}\right|\right) \leq \sum_{i=1}^{M} \underline{r}^{i}\left(M\left|\mathcal{X}_{t}\right|\right)$ and hence

$$
\underline{r}^{i}\left(\left|\mathcal{X}_{t}\right| / M\right) \leq \underline{r}^{i}\left(\frac{1}{M} \sum_{i=1}^{M}\left|x_{t}^{i}\right|\right) \leq \sum_{i=1}^{M} \underline{r}^{i}\left(\left|x_{t}^{i}\right|\right) .
$$

${ }^{3}$ It is worth noting that, instead of the above definition of $V$, a weighted sum of Lyapunov functions could be used along the reasoning provided in [34] in the framework of composite systems. 
Therefore, letting $\underline{r}\left(\left|\mathcal{X}_{t}\right|\right) \triangleq \underline{r}^{i}\left(\left|\mathcal{X}_{t}\right| / M\right)$ for an arbitrarily chosen index $i$, we showed that

$$
\begin{gathered}
\underline{r}\left(\left|\mathcal{X}_{t}\right|\right) \leq V\left(\mathcal{X}_{t}, \overline{\mathcal{W}}_{t}\right), \quad \forall \mathcal{X}_{t} \in \mathscr{X}, \forall \overline{\mathcal{W}}_{t} \in \mathscr{W}, \\
V\left(\mathcal{X}_{t}, \overline{\mathcal{W}}_{t}\right) \leq \beta\left(\left|\mathcal{X}_{t}\right|\right)+\sigma\left(\left|\overline{\mathcal{W}}_{t}\right|\right), \\
\forall \mathcal{X}_{t} \in \mathscr{X}_{f}, \forall \overline{\mathcal{W}}_{t} \in \mathscr{W}
\end{gathered}
$$

From (22), defining

$$
\Delta V \triangleq \sum_{i=1}^{M} V^{i}\left(x_{t+1}^{i}, w_{t+1}^{i}\right)-\sum_{i=1}^{M} V^{i}\left(x_{t}^{i}, w_{t}^{i}\right),
$$

it follows that

$$
\Delta V \leq-\sum_{i=1}^{M} \underline{r}^{i}\left(\left|x_{t}\right|\right)+\sum_{i=1}^{M} \varphi_{1}^{i}\left(\left|w_{t}^{i}\right|\right)+\sum_{i=1}^{M} \varphi_{2}^{i}\left(\left|w_{t+1}^{i}\right|\right) .
$$

Moreover

$$
\begin{gathered}
-\sum_{i=1}^{M} \underline{r}^{i}\left(\left|x_{t}\right|\right) \leq-\underline{r}^{i}\left(\left|\mathcal{X}_{t}\right| / M\right), \\
\sum_{i=1}^{M} \varphi_{1}^{i}\left(\left|w_{t}^{i}\right|\right) \leq \sum_{i=1}^{M} \varphi_{1}^{i}\left(\left|\overline{\mathcal{W}}_{t}\right|\right),
\end{gathered}
$$

and

$$
\sum_{i=1}^{M} \varphi_{2}^{i}\left(\left|w_{t+1}^{i}\right|\right) \leq \sum_{i=1}^{M} \varphi_{2}^{i}\left(\left|\overline{\mathcal{W}}_{t+1}\right|\right) .
$$

Then, letting

$$
\varphi_{1}\left(\left|\overline{\mathcal{W}}_{t}\right|\right) \triangleq \sum_{i=1}^{M} \varphi_{1}^{i}\left(\left|W_{t}^{i}\right|\right)
$$

and

$$
\varphi_{2}\left(\left|\overline{\mathcal{W}}_{t+1}\right|\right) \triangleq \sum_{i=1}^{M} \varphi_{2}^{i}\left(\left|W_{t+1}^{i}\right|\right),
$$

it follows that

$$
\begin{aligned}
\Delta V & \leq-\underline{r}\left(\left|\mathcal{X}_{t}\right|\right)+\varphi_{1}\left(\left|\overline{\mathcal{W}}_{t}\right|\right)+\varphi_{2}\left(\left|\overline{\mathcal{W}}_{t+1}\right|\right) \\
& \leq-\underline{r}\left(\left|\mathcal{X}_{t}\right|\right)+\varphi_{t o t}\left(|| \overline{\mathcal{W}}_{[t+1]}||\right), \\
\forall \mathcal{X}_{t} & \in \mathscr{X}, \forall \overline{\mathcal{W}} \in \mathcal{M}_{\mathscr{W}},
\end{aligned}
$$

where $\varphi_{t o t}(s) \triangleq \varphi_{1}(s)+\varphi_{2}(s)$. Therefore, by (26), (27) and (28), $V\left(\mathcal{X}_{t}, \overline{\mathcal{W}}_{t}\right)$ is an ISS-Lyapunov function in $\mathscr{X}^{M P C}$ for system (24) and hence this system is ISS in $\mathscr{X}^{M P C}$.

As far as system (25) is concerned (we recall that this system describes the effects of the time-delays in the information exchange variables), the proof that it is ISS is obviously trivial since (25) is an asymptotically stable discrete-time linear system. We only very briefly sketch some parts of the proof just for the purpose of introducing a few quantities that will be used subsequently. Following [30], a candidate ISSLyapunov function for system (25) is $V^{D}\left(\mathcal{Z}_{t}\right) \triangleq \mathcal{Z}_{t}^{\top} P \mathcal{Z}_{t}$, where $P$ is the positive definite solution of the Lyapunov equation $A^{\top} P A-P=-Q$ for a given symmetric positivedefinite matrix $Q$. It is immediate to show that

$$
\underline{r}^{D}\left(\left|\mathcal{Z}_{t}\right|\right) \leq V^{D}\left(\mathcal{Z}_{t}\right) \leq \beta^{D}\left(\left|\mathcal{Z}_{t}\right|\right)
$$

where $\underline{r}^{D}(s) \triangleq \lambda_{\min }(P) s^{2}$ and $\beta^{D}(s) \triangleq \lambda_{\max }(P) s^{2}$ $\left(\lambda_{\min }(P)\right.$ and $\lambda_{\max }(P)$ denote the minimum and maximum eigenvalues of $P$, respectively). Moreover, defining $\Delta V^{D} \triangleq$ $V^{D}\left(\mathcal{Z}_{t+1}\right)-V^{D}\left(\mathcal{Z}_{t}\right)$ it is straightforward to obtain

$$
\begin{gathered}
\Delta V^{D} \leq-\underline{\underline{r}}^{D}\left(\left|\mathcal{Z}_{t}\right|\right)+\varphi_{1}^{D}\left(\left|\mathcal{X}_{t}\right|\right) \\
\text { where } \quad \underline{\tilde{r}}^{D}(s) \triangleq \quad \text { and } \\
\varphi_{1}^{D}(s) \triangleq \lambda_{\max }\left(\frac{2 B^{\top} P A A^{\top} P B}{\lambda_{\min }(Q)}+B^{\top} P B\right) s^{2} .
\end{gathered}
$$

Now, recalling from (25) that $\overline{\mathcal{W}}_{t}=C \mathcal{Z}_{t}$, from the proof of Lemma 4.2, from (28) it follows immediately that the ISSLyapunov function $V\left(\mathcal{X}_{t}, \overline{\mathcal{W}}_{t}\right)$ satisfies

$$
\begin{aligned}
& V\left(\mathcal{X}_{t+1}, \overline{\mathcal{W}}_{t+1}\right)-V\left(\mathcal{X}_{t}, \overline{\mathcal{W}}_{t}\right) \\
& \leq-\alpha_{4}\left(V\left(\mathcal{X}_{t}, \overline{\mathcal{W}}_{t}\right)\right)+\varphi_{1}\left(\left|\overline{\mathcal{W}}_{t}\right|\right)+\varphi_{2}\left(|| \overline{\mathcal{W}}_{t+1} \mid\right) \\
& \leq-\alpha_{4}\left(V\left(\mathcal{X}_{t}, \overline{\mathcal{W}}_{t}\right)\right)+\varphi_{w}\left(V^{D}\left(\mathcal{Z}_{t}\right)\right) \\
&+\varphi_{w+1}\left(V^{D}\left(\mathcal{Z}_{t+1}\right)\right) \\
& \leq-\alpha_{4}\left(V\left(\mathcal{X}_{t}, \overline{\mathcal{W}}_{t}\right)\right)+\varphi_{w_{t o t}}\left(\| V^{D}(\mathcal{Z})_{[t+1]}||\right), \\
& \forall \mathcal{X}_{t} \in \mathscr{X}_{f}, \forall \mathcal{Z} \in \mathcal{M}_{\mathbb{R}^{n} \mathcal{Z}}
\end{aligned}
$$

where $\alpha_{4}$ is defined as in the proof of Theorem 4.2, whereas $\varphi_{11} \triangleq \varphi_{1}+\varepsilon, \varphi_{w} \triangleq \varphi_{1} \circ\left(\underline{r}^{D}\right)^{-1}, \varphi_{w+1} \triangleq \varphi_{2} \circ\left(\underline{r}^{D}\right)^{-1}$ and $\varphi_{w_{t o t}}(s) \triangleq \varphi_{w}(s)+\varphi_{w+1}(s)$. Moreover, as far as the $I S S$-Lyapunov function $V^{D}\left(\mathcal{Z}_{t}\right)$ is concerned, from (29) it follows that

$$
\Delta V^{D} \leq-\alpha_{4}^{D}\left(V^{D}\left(\mathcal{Z}_{t}\right)\right)+\varphi_{w}^{D}\left(V\left(\mathcal{X}_{t}, \overline{\mathcal{W}}_{t}\right)\right)
$$

where, again, $\alpha_{4}^{D}$ is defined analogously to the above definition of $\alpha_{4}$, whereas $\varphi_{w}^{D} \triangleq \varphi_{1}^{D} \circ\left(\underline{r}^{D}\right)^{-1}$.

Analogously to the proof of Theorem 4.1, given $e \in \mathbb{R}_{\geq 0}$, let $R(e) \triangleq\{\mathcal{X}: V(\mathcal{X}, \overline{\mathcal{W}}) \leq e, \forall \overline{\mathcal{W}} \in \mathscr{W}\}$. Let $\Theta \triangleq\{\mathcal{X}:$ $\left.V(\mathcal{X}, \overline{\mathcal{W}}) \leq \bar{e}=\max _{R(e) \subseteq \mathscr{X}_{f}} e, \bar{\forall} \overline{\mathcal{W}} \in \mathscr{W}\right\}$. Note that $\bar{e}>b(\hat{\mathcal{W}})$ and $D \subset \Theta$. Since the region $D$ is reached asymptotically, the state will arrive in $\Theta$ in a finite time, that is, there exists $T_{\theta}$ such that $V\left(\mathcal{X}_{k}, \overline{\mathcal{W}}_{k}\right) \leq \bar{e}, \forall k \geq T_{\theta}$. Hence, the region $\Theta$ is a robust positively invariant set for the system (1). Thanks to Remark 3.7 in [30], from (30) and (31) it follows that there exist some $\mathcal{K} \mathcal{L}$-functions $\hat{\beta}$ and $\hat{\beta}^{D}$ such that

$$
\begin{gathered}
V\left(\mathcal{X}_{k}, \overline{\mathcal{W}}_{k}\right) \leq \max \left\{\hat{\beta}\left(V\left(\mathcal{X}_{t}, \overline{\mathcal{W}}_{t}\right), k\right), \gamma_{1}\left(\left\|V^{D}(\mathcal{Z})_{[k]}\right\|\right)\right\} \\
\forall \mathcal{X}_{t} \in \Theta, \forall k \in \mathbb{Z}_{\geq 0}, k \geq t \\
V^{D}\left(\mathcal{Z}_{k}\right) \leq \max \left\{\hat{\beta}^{D}\left(V^{D}\left(\mathcal{Z}_{t}\right), k\right), \gamma_{2}\left(\left\|V(\mathcal{X}, \overline{\mathcal{W}})_{[k]}\right\|\right)\right\} \\
\forall \mathcal{X} \in \mathscr{X}, \forall k \in \mathbb{Z}_{\geq 0}, k \geq t
\end{gathered}
$$

where we define

$$
\gamma_{1} \triangleq \alpha_{4}^{-1} \circ \rho^{-1} \circ \varphi_{w_{t o t}}
$$

and

$$
\gamma_{2} \triangleq\left(\alpha_{4}^{D}\right)^{-1} \circ \rho^{-1} \circ \varphi_{w}^{D},
$$

with $\rho$ any $\mathcal{K}_{\infty}$-function such that $(\operatorname{Id}-\rho) \in \mathcal{K}_{\infty}$.

Now, the following result about the stability properties of the team of cooperating agents can be proved. The proof is a slight modification of the one in [30] and will be reported here for the sake of clarity and completeness. 
Theorem 4.3: Suppose that Assumptions 1-4 are verified. Moreover, assume that the following small gain condition holds:

$$
\gamma_{1} \circ \gamma_{2}(s)<s .
$$

with $\gamma_{1}$ and $\gamma_{2}$ given by (34) and (35) and argument $s$ takes its values from a suitable subset of $\mathbb{R}_{\geq 0}$ according to inequalities (30)-(33). Then the team of cooperating agents described by the interconnected dynamic equations (24) and (25) is 0-AS in $\mathscr{X}^{M P C} \times \mathbb{R}^{n_{\mathcal{Z}}}$. that

Proof: If $\gamma_{1} \circ \gamma_{2}(s)<s$, from (32) and (33) it follows

$$
\begin{aligned}
& V\left(\mathcal{X}_{k}, \overline{\mathcal{W}}_{k}\right) \leq \max \left\{\hat{\beta}\left(V\left(\mathcal{X}_{t}, \overline{\mathcal{W}}_{t}\right), t\right),\right. \\
&\left.\gamma_{1}\left(\hat{\beta}^{D}\left(V^{D}\left(\mathcal{Z}_{t}\right), t\right)\right)\right\}, \\
& \forall \mathcal{X}_{t} \in \Theta, \forall k \in \mathbb{Z}_{\geq 0}, k \geq t \\
& V^{D}\left(\mathcal{Z}_{k}\right) \leq \max \left\{\hat{\beta}^{D}\left(V^{D}\left(\mathcal{Z}_{t}\right), t\right),\right. \\
&\left.\gamma_{2}\left(\hat{\beta}\left(V\left(\mathcal{X}_{t}, \overline{\mathcal{W}}_{t}\right), t\right)\right)\right\}, \\
& \forall \mathcal{X}_{t} \in \Theta, \forall k \in \mathbb{Z}_{\geq 0}, k \geq t
\end{aligned}
$$

and hence $V\left(\mathcal{X}_{k}, \overline{\mathcal{W}}_{k}\right), V^{D}\left(\mathcal{Z}_{k}\right)$ are bounded by initial condition. By Lemma 3.13 in [30], an asymptotic gain from $V^{D}\left(\mathcal{Z}_{k}\right)$ to $V\left(\mathcal{X}_{k}, \overline{\mathcal{W}}_{k}\right)$ is given by $\gamma_{1}$ whereas an asymptotic gain from $V\left(\mathcal{X}_{k}, \overline{\mathcal{W}}_{k}\right)$ to $V^{D}\left(\mathcal{Z}_{k}\right)$ is given by $\gamma_{2}$. Hence:

$$
\begin{aligned}
\varlimsup_{k \rightarrow \infty} V\left(\mathcal{X}_{k}, \overline{\mathcal{W}}_{k}\right) \leq & \varlimsup_{k \rightarrow \infty}\left[\alpha_{4}^{-1} \circ \rho^{-1} \circ \varphi_{w}\left(V^{D}\left(\mathcal{Z}_{k-1}\right)\right)\right. \\
& \left.+\alpha_{4}^{-1} \circ \rho^{-1} \circ \varphi_{w+1}\left(V^{D}\left(\mathcal{Z}_{k}\right)\right)\right], \\
\leq & \alpha_{4}^{-1} \circ \rho^{-1} \circ \varphi_{w}\left(\lim _{k \rightarrow \infty} V^{D}\left(\mathcal{Z}_{k-1}\right)\right) \\
& +\alpha_{4}^{-1} \circ \rho^{-1} \circ \varphi_{w+1}\left(\lim _{k \rightarrow \infty} V^{D}\left(\mathcal{Z}_{k}\right)\right) .
\end{aligned}
$$

But

$$
\varlimsup_{k \rightarrow \infty} V^{D}\left(\mathcal{Z}_{k-1}\right)=\varlimsup_{k \rightarrow \infty} V^{D}\left(\mathcal{Z}_{k}\right)
$$

Hence

$$
\begin{aligned}
\varlimsup_{k \rightarrow \infty} V\left(\mathcal{X}_{k}, \overline{\mathcal{W}}_{k}\right) & \leq \alpha_{4}^{-1} \circ \rho^{-1} \circ \varphi_{w_{t o t}}\left(\varlimsup_{k \rightarrow \infty} V^{D}\left(\mathcal{Z}_{k}\right)\right) \\
& =\gamma_{1}\left(\varlimsup_{k \rightarrow \infty} V^{D}\left(\mathcal{Z}_{k}\right)\right), \\
& \leq \gamma_{1} \circ \gamma_{2}\left(\overline{\lim }_{k \rightarrow \infty} V\left(\mathcal{X}_{k}, \overline{\mathcal{W}}_{k}\right)\right) .
\end{aligned}
$$

Again, the assumption that $\gamma_{1} \circ \gamma_{2}(s)<s$ implies that

$$
\varlimsup_{k \rightarrow \infty} V^{D}\left(\mathcal{Z}_{k}\right)=\varlimsup_{k \rightarrow \infty} V\left(\mathcal{X}_{k}, \overline{\mathcal{W}}_{k}\right)=0 .
$$

Thus, the system is $0-A S$ in $\mathscr{X}^{M P C} \times \mathbb{R}^{n \mathcal{Z}}$.

Remark 4.1: It is worth noting that the small-gain condition (36) may turn out to be conservative in practice as it is typical of these kind of results. In this respect, it is important to cite the results by F. Wirth and co-workers [35] where vector small-gain theorems have been presented). On the other hand, the generality of the problem makes it rather difficult to obtain tighter conditions without introducing more restrictive assumptions on the structure of the agents' dynamics and on the cost function. Indeed, for special classes of cooperative control problems, different conditions for the stability of the team of agents can be obtained. For instance, we recall that in [14] stability has been shown for formation control of UAV's under different hypotheses as the knowledge of the neighbors dynamics, suitably fast information exchange and bounded error between the predicted and actuated state trajectories of each member of the team. As another example, stability of a set of decoupled systems is ensured in [21], by assuming the knowledge of feasibility regions and a specific hierarchical design of the decentralized RH control problem: the computations are shared by nodes with different priorities, which can impose their control decisions on the subordinate neighbors.

Remark 4.2: As expected, in the special case where the state equation (4) takes on a linear structure, the FH cost function (9) is quadratic, and no state and control constraints are present, more specialized and tight results can be found. In particular, the control law takes on an explicit feedbackfeedforward structure and some interesting properties hold. The reader is referred to [36] for more details.

\section{SimUlation RESUlTS}

In this section we will show some simulation results concerning a team of UAVs moving in $\mathbb{R}^{2}$ with nonlinear dynamics. Such a problem has been selected because of its reasonable simplicity so as to be able to ascertain the basic features and properties of the proposed cooperative control law. A team of $M=3$ vehicles will be considered, whose continuous-time models and data are taken according to [37]:

$$
\begin{aligned}
& m \ddot{x}^{i}=-\mu_{1} \dot{x}^{i}+\left(u_{R}^{i}+u_{L}^{i}\right) \cos \left(\theta^{i}\right), \\
& m \ddot{y}^{i}=-\mu_{1} \dot{y}^{i}+\left(u_{R}^{i}+u_{L}^{i}\right) \sin \left(\theta^{i}\right), \\
& J \ddot{\theta}^{i}=-\mu_{2} \dot{\theta}^{i}+\left(u_{R}^{i}-u_{L}^{i}\right) r_{v} .
\end{aligned}
$$

where $i=1,2,3$. For simplicity, we assume that all the members of the team have the same physical parameters: the mass is $m=0.75 \mathrm{Kg}$, the inertia is $J=0.00316 \mathrm{Kgm}^{2}$, the linear friction coefficient is $\mu_{1}=0.15 \mathrm{Kg} / \mathrm{s}$ and the rotational friction coefficient is $\mu_{2}=0.005 \mathrm{Kgm}^{2} / \mathrm{s}$ and finally the radius of the vehicle is $r_{v}=8.9 \mathrm{~cm}$. The state vector of each agent will be from now on denoted as $z^{i}$, and is defined by considering the position and velocity in each direction of the plane, plus the orientation angle and rotational velocity $z^{i} \triangleq \operatorname{col}\left(\theta^{i}, \dot{\theta}^{i}, x^{i}, \dot{x}^{i}, y^{i}, \dot{y}^{i}\right)$, whereas the control vector is given by $u^{i} \triangleq \operatorname{col}\left(u_{L}^{i}, u_{R}^{i}\right)$. The continuous-time models (37) are discretized with a sampling time $T=0.1 \mathrm{~s}$, thus obtaining suitable discrete-time models, where the state vectors are denoted by $z_{t}^{i}$ and the control vectors are denoted by $u_{t}^{i}$.

Remark 5.1: In the following, the simulation trials will refer to the above approximated discrete-time model for mere illustration purposes and to show the effectiveness of the proposed cooperative control scheme. However, as shown in [38], in some cases the control law that stabilizes the approximated discrete-time model may perform quite poorly when applied to the exact model. This is clearly an important issue and we refer the reader to the above reference for more details and to the works [39], [40] for the general case of control of nonlinear sampled-data systems. For a MPC algorithm where 
the continuous time evolution of the system is explicitly taken into account, while the optimization is performed with respect to a piece-wise constant control signal, see [16].

The objective of the distributed cooperative controller is to reach a certain formation following a predefined desired trajectory, based on leader one, for each UAV. The desired trajectories have been chosen with constant velocities and null rotational velocity. At every time instant $t$, each agent solves a local Problem 3.1 with FH cost function

$$
\begin{aligned}
J_{F H}^{i}= & \sum_{l=t}^{t+N_{p}^{i}-1}\left(\left\|z_{l}^{i}-\bar{z}_{l}^{1}+d^{i 1}\right\|_{Q^{i}}^{2}+\left\|u_{l}^{i}-\bar{u}^{i}\right\|_{R^{i}}^{2}\right) \\
& +\left\|z_{t+N_{p}^{i}}^{i}-\bar{z}_{t+N_{p}^{i}}^{1}+d^{i 1}\right\|_{P^{i}}^{2} \\
& +\sum_{l=t}^{t+N_{p}^{i}-1} \sum_{j \in G^{i}}\left\|z_{l}^{i}-\tilde{z}_{l}^{j}+d^{i j}\right\|_{S^{i j}}^{2}
\end{aligned}
$$

where $\bar{z}_{l}^{1}$ represents the desired trajectory of the leader while $d^{i j}$ are the desired distance between agent $i$ and agent $j\left(d^{i i}=\right.$ $0, \forall i=1, \ldots, M)$. Hence the term $\bar{z}_{l}^{1}-d^{i 1}$ represents the desired trajectory of the $i-t h \mathrm{UAV}$. The values of $d^{i j}$ are such that the three UAVs assume a triangle formation. The term $\bar{u}^{i}$ is the control vector necessary in order to maintain each UAV on the desired trajectory. For the information vector to take on a constant value within the prediction horizon, we let

$$
\tilde{z}_{t+k}^{j}=\left(\bar{z}_{t+k}^{1}-d^{j 1}\right)+\left(z_{t-\Delta^{i j}}^{j}-\bar{z}_{t-\Delta^{i j}}^{1}+d^{j 1}\right) .
$$

The delays have all been set to $\Delta^{i j}=\Delta=5 T$ and the communication topology is assumed to be stationary. Specifically, we suppose that the leader does not receive any information from the other agents (hence $S^{1 j}=0, \forall j \in G^{1}$ ). Moreover agent 2 gets information from the leader and from agent 3 and, analogously, agent 3 gets information from the leader and from agent 2 .

The values of the parameters used for the leader are $N_{c}^{1}=$ $N_{p}^{1}=5, Q^{1}=0.1 \cdot \operatorname{diag}(1,50,1,1,1,1), R^{1}=0.01$. $\operatorname{diag}(1,1)$, and $S^{1 j}=0, \forall j \in G^{1}$. The lengths of horizons $N_{c}^{1}, N_{p}^{1}$, though quite small, are indeed sufficient for the leader to show a reasonably good tracking performance as it starts quite close to the desired trajectory. For the other agents, we consider the same values of the parameters, that is, we have $N_{c}^{i}=10, N_{p}^{i}=250, Q^{i}=0.1 \cdot \operatorname{diag}(1,50,1,1,1,1)$, $R^{i}=0.01 \cdot \operatorname{diag}(1,1), S^{i j}=\operatorname{diag}(0.1,0.1,1,0.1,1,0.1)$, $\alpha_{w}=0.96, i=2,3$. The matrices $P^{i}$ are obtained, from the choice of $Q^{i}, R^{i}$ and $S^{i j}$, by the auxiliary control law designed according to Lemma 4.1 using $\beta^{i}=3$ and $\tilde{S}^{i j}=2 S^{i j}, i=$ $1,2,3$. The FH Optimal Control Problem 3.1 is characterized by the constraints $u_{\text {Lmin }}^{i} \leq u_{t}^{1 i} \leq u_{R \max }^{i} ; u_{\text {Lmin }}^{i} \leq u_{t}^{2 i} \leq$ $u_{R \max }^{i}$, with $u_{L \min }^{i}=0, u_{L \max }^{i}=6, u_{R \min }^{i}=0$, and $u_{R \max }^{i}=6, \quad i \stackrel{\max }{=} 1,2,3$, where $u_{t}^{1 i}\left(u_{t}^{2 t}\right)$ denotes the first (second) component of vector $u_{t}^{i}$. Moreover, the terminal constraints $\left\|z_{t+N_{p}^{i}}^{i}-\bar{z}_{t+N_{p}^{i}}^{1}+d^{i 1}\right\|_{P^{i}}^{2} \leq \Upsilon^{i}, i=1,2,3$, have been obtained numerically according to Lemma 4.1. The values of $\Upsilon^{i}$ are constant along the trajectories and are respectively $\Upsilon^{1}=0.3$ and $\Upsilon^{i}=1.2, \quad i=2,3$. These values are not comparable since the matrices $P^{i}$ are different. The control necessary in order to maintain each UAV on the desired trajectory is $\bar{u}^{1 i}=1, \bar{u}^{2 i}=1$. The values of the desired distances between the agents are the following:

$$
\begin{aligned}
d^{12}= & 16 \operatorname{col}(0,0,-\sin (\pi / 3) \cos (\pi / 4)-0.5 \cos (\pi / 4), 0, \\
& -\sin (\pi / 3) \cos (\pi / 4)+0.5 \cos (\pi / 4), 0) \\
d^{13}= & 16 \operatorname{col}(0,0,-\sin (\pi / 3) \cos (\pi / 4)+0.5 \cos (\pi / 4), 0, \\
& -\sin (\pi / 3) \cos (\pi / 4)-0.5 \cos (\pi / 4), 0) \\
d^{21}= & 16 \operatorname{col}(0,0,+\sin (\pi / 3) \cos (\pi / 4)+0.5 \cos (\pi / 4), 0, \\
& +\sin (\pi / 3) \cos (\pi / 4)-0.5 \cos (\pi / 4), 0), \\
d^{23}= & 16 \operatorname{col}(0,0, \cos (\pi / 4), 0,-\cos (\pi / 4), 0) \\
d^{31}= & 16 \operatorname{col}(0,0, \sin (\pi / 3) \cos (\pi / 4)-0.5 \cos (\pi / 4), 0, \\
& +\sin (\pi / 3) \cos (\pi / 4)+0.5 \cos (\pi / 4), 0), \\
d^{32}= & 16 \operatorname{col}(0,0,-\cos (\pi / 4), 0, \cos (\pi / 4), 0)
\end{aligned}
$$

Moreover, the initial condition of the desired trajectory of the leader is:

$$
\begin{aligned}
\bar{z}_{0}^{1}= & \operatorname{col}\left(\pi / 4,0,0, \frac{1}{m}\left(\bar{u}^{1 i}+\bar{u}^{2 i}\right) \cos (\pi / 4), 0,\right. \\
& \left.\frac{1}{m}\left(\bar{u}^{1 i}+\bar{u}^{2 i}\right) \sin (\pi / 4)\right) .
\end{aligned}
$$

The entire desired leader's trajectory is obtained, starting from the initial conditions, holding constant the velocities. Finally, the initial conditions of the UAVs are $z_{0}^{1}=\bar{z}_{0}^{1}, z_{0}^{2}=\bar{z}_{0}^{2}, z_{0}^{3}=$ $\bar{z}_{0}^{2}+3.8 d^{23}$.

In Fig. 2, the team trajectories are reported in the twodimensional space: the objective is to attain a triangle formation along a straight line of $45^{\circ}$ as followers of the leader. The dotted lines depict the actual behavior of the agents. It is worth noting the cooperative behavior of the agents: in particular agent 2 (on the left of the leader) even if it starts on its trajectory $\left(z_{0}^{2}=\bar{z}_{0}^{2}\right)$ it moves on the right in order to reach faster a better (with respect to cost (38)) formation with agent 3 . Without the cooperative term in the cost function the trajectory of agent 2 would be a straight line.

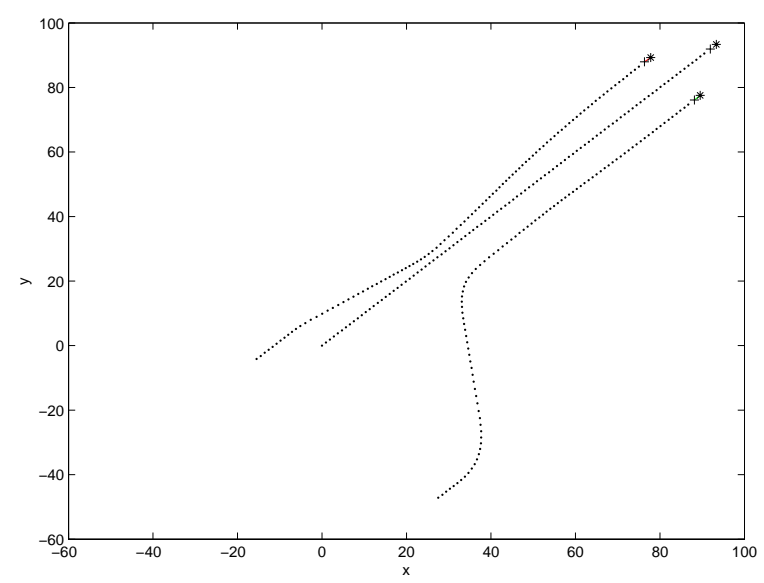

Fig. 2. Team trajectories (dotted lines). The front of the vehicle is represented by the symbol '*' whereas the back of the vehicle is represented by the symbol '+'.

In Fig. 3, the behaviors of the control variables $u^{i}=$ $\operatorname{col}\left(u_{L}^{i}, u_{R}^{i}\right)$ of Agents 2 and 3 are shown. In particular, in Figs. 3(a) and 3(b) the behaviors of the first component $u_{L}^{i}$ of the control variables are plotted, whereas in Figs. 3(c) and 3(d), the differences $u_{L}^{i}-u_{R}^{i}$ between the first and the second 
components of the control variables are shown. This has been done to better appreciate the differences between the first and the second components of the control variables; actually, these differences are rather small due to the small magnitude of the variations of the orientation of the two agents. In Fig. 3, the dashed lines depict the constraints imposed on the control variables.
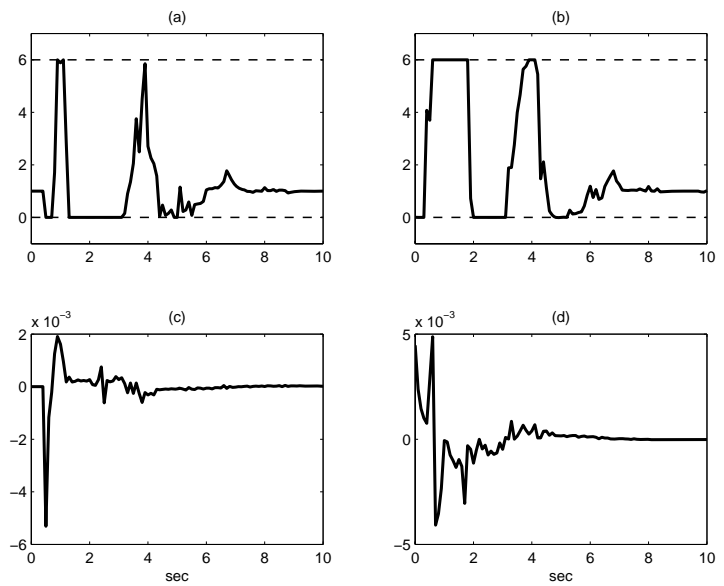

Fig. 3. Behaviors of the control variables of Agents 2 and 3. (a) and (b) behaviors of the first component $u_{L}^{i}$ of the control variables. (c) and (d) difference $u_{L}^{i}-u_{R}^{i}$ between the first and the second components of the control variables. Dashed lines: control constraints.

\section{Conclusions}

In this paper, we investigate the problem of designing cooperative control algorithms for a team of distributed agents with nonlinear discrete-time dynamics, and analyzing the stability properties of the overall closed-loop system. The problem formulation is based on a decentralized RH control framework, where the dynamics of the distributed agents are linked by a cooperative cost function. Each agent uses locally computed control laws, which take into consideration delayed state information from neighboring agents. The resulting local control laws take the form of a feedback-feedforward structure, which is derived by a nonlinear $\mathrm{RH}$ framework.

A key contribution is the general problem formulation, which allows the systematic derivation of rigorous stability results. The stability analysis is made possible by combining the stability properties of the RH local control laws and ISS arguments. Finally, the team of cooperating agents is treated as a single dynamical system resulting from a feedback interconnection of regionally ISS systems, thus allowing the use of small-gain conditions to show asymptotic stability.

Despite the general formulation, there are some important issues requiring further investigation. Future research efforts will be devoted towards (i) considering the case where disturbances and uncertainties affect the communication between the agents of the team and (ii) addressing the robustness issue by generalizing the methodology to the case where optimality of the algorithm is not required at each time-stage, and (iii) considering less conservative small-gain conditions (like, for instance, vector small-gain results).

\section{APPENDIX}

Proof of Lemma 4.1. Owing to the smoothness of $\kappa_{f}^{i}\left(x^{i}\right)$ and the fact that it is a stabilizing control law and recalling that $0 \in X^{i}, 0 \in U^{i}$, it follows that there exists $\Upsilon_{1}^{i} \in(0, \infty)$ such that Points 1,2 , and 3 of Assumption 1 are satisfied for $x^{i^{\top}} \Pi^{i} x^{i} \leq \Upsilon_{1}^{i}$. Point 5 is satisfied with $\alpha_{h_{f}^{i}}\left(\left|x^{i}\right|\right)=\lambda_{\min }\left(\Pi^{i}\right)\left|x^{i}\right|^{2}$, and $\beta_{h_{f}^{i}}\left(\left|x^{i}\right|\right)=\lambda_{\max }\left(\Pi^{i}\right)\left|x^{i}\right|^{2}$, where $\lambda_{\min }\left(\Pi^{i}\right)$ and $\lambda_{\max }\left(\Pi^{i}\right)$ denote the minimum and the maximum eigenvalues of $\Pi^{i}$, respectively. In order to prove Point 6, letting

$$
\Phi^{i}\left(x^{i}\right) \triangleq f^{i}\left(x^{i}, K^{i} x^{i}\right)-A_{c l}^{i} x^{i},
$$

the inequality

$$
\begin{aligned}
f^{i}\left(x^{i},\right. & \left.K^{i} x^{i}\right)^{\top} \Pi^{i} f^{i}\left(x^{i}, K^{i} x^{i}\right)-x^{i^{\top}} \Pi^{i} x^{i} \\
\leq & -x^{i^{\top}}\left(Q^{i}+K^{i^{\top}} R^{i} K^{i}\right) x^{i}-q^{i}\left(x^{i}, w^{i}\right) \\
& +\psi^{i}\left(\left|w^{i}\right|\right)
\end{aligned}
$$

is equivalent to

$$
\begin{aligned}
& 2 \Phi^{i}\left(x^{i}\right)^{\top} \Pi^{i} A_{c l}^{i} x^{i}+\Phi^{i}\left(x^{i}\right)^{\top} \Pi^{i} \Phi^{i}\left(x^{i}\right)+x^{\top} A_{c l}^{i^{\top}} \Pi^{i} A_{c l}^{i} x^{i} \\
& -x^{i^{\top}} \Pi^{i} x^{i} \leq-x^{i^{\top}}\left(Q^{i}+K^{i^{\top}} R^{i} K^{i}\right) x^{i}-q^{i}\left(x^{i}, w^{i}\right) \\
& +\psi^{i}\left(\left|w^{i}\right|\right) .
\end{aligned}
$$

Indeed, from (23) it is easy to show that inequality (40) is equivalent to

$$
\begin{aligned}
& 2 \Phi^{i}\left(x^{i}\right)^{\top} \Pi^{i} A_{c l}^{i^{\top}} x^{i}+\Phi^{i}\left(x^{i}\right)^{\top} \Pi^{i} \Phi^{i}\left(x^{i}\right) \\
& \leq x^{i^{\top}} \tilde{Q}^{i} x^{i}-x^{i^{\top}}\left(Q^{i}+K^{i^{\top}} R^{i} K^{i}\right) x^{i}-q^{i}\left(x^{i}, w^{i}\right) \\
& \quad+\psi^{i}\left(\left|w^{i}\right|\right) .
\end{aligned}
$$

Now, define $L_{r}^{i} \triangleq \sup _{x^{i} \in B_{r}^{i}}\left|\Phi^{i}\left(x^{i}\right)\right| /\left|x^{i}\right|$, where $B_{r}^{i} \triangleq$ $\left\{x^{i}:\left|x^{i}\right| \leq r\right\}$ (Once chosen $r, L_{r}^{i}$ does exist and takes on a finite value because $\left.f^{i} \in \mathscr{C}^{2}\right)$. Moreover by the assumption on $q^{i}\left(x^{i}, w^{i}\right)$ and the definition of $\tilde{Q}$ it follows that

$$
\begin{aligned}
& x^{i^{\top}} \tilde{Q}^{i} x^{i}-x^{i^{\top}}\left(Q^{i}+K^{i^{\top}} R^{i} K^{i}\right) x^{i}-q^{i}\left(x^{i}, w^{i}\right) \\
& +\psi^{i}\left(\left|w^{i}\right|\right) \geq x^{i^{\top}} \tilde{Q}^{i} x^{i}-x^{i^{\top}}\left(Q^{i}+K^{i^{\top}} R^{i} K^{i}\right) x^{i} \\
& -x^{i^{\top}} \tilde{S}_{i} x^{i^{\top}} \geq \gamma^{i}\left|x^{i}\right|^{2}, \quad \gamma^{i}>0 .
\end{aligned}
$$

Then, $\forall x^{i} \in B_{r}^{i}$, (41) is satisfied if

$$
\gamma^{i}\left|x^{i}\right|^{2} \geq\left\{2 L_{r}^{i}\left|\Pi^{i}\right|\left|A_{c l}^{i}\right|+L_{r}^{i^{2}}\left|\Pi^{i}\right|\right\}\left|x^{i}\right|^{2} .
$$

Hence, since $L_{r}^{i} \rightarrow 0$ as $r \rightarrow 0$, there exists $\Upsilon^{i} \in\left(0, \Upsilon_{1}^{i}\right)$ such that inequality (42) holds $\forall x^{i} \in X_{f}^{i}$, which implies that inequality (39) holds as well. Finally, there exists $\bar{N}_{p}$ such that for all $N_{p} \geq \bar{N}_{p}, \forall x^{i} \notin X_{f}^{i}, \forall w^{i} \in W_{i}$

$$
\begin{aligned}
& h_{f}^{i}\left(f^{i}\left(x^{i}, \kappa_{f}^{i}\left(x^{i}\right)\right)\right)-h_{f}^{i}\left(x^{i}\right) \\
& \quad \leq-x^{i^{\top}}\left(Q^{i}+K^{i^{\top}} R^{i} K^{i}\right) x^{i} \\
& \quad-q^{i}\left(x^{i}, \tilde{w}^{i}\right)+\psi^{i}\left(\left|\tilde{w}^{i}\right|\right), \\
& \quad<0,
\end{aligned}
$$


where $\tilde{w}^{i}=\left(A_{w}^{i}\right)^{N_{p}^{i}-1} w^{i}$ so that Point 4 of Assumption 1 is satisfied, too, thus ending the proof.

\section{ACKNOWLEDGMENT}

The authors are grateful to the Associate Editor and to the anonymous Reviewers for their valuable and constructive criticisms and suggestions, and to Dr. Gilberto Pin for several useful comments.

\section{REFERENCES}

[1] S. H. Wang and E. Davidson, "On the stabilization of decentralized control systems," IEEE Transactions on Automatic Control, vol. 18 , no. 5, pp. 473-478, 1973.

[2] D. D. Siljak, Large-Scale Dynamic Systems. New York: North-Holland, 1978.

[3] P. Ioannou, "Decentralized adaptive control of interconnected systems," IEEE Trans. on Automatic Control, vol. 31, no. 4, pp. 291 - 298, 1986.

[4] E. Camponogara, D. Jia, B. H. Krogh, and S. Talukdar, "Distributed model predictive control," IEEE Control System Magazine, vol. 22, no. 1, pp. 44-52, February 2002.

[5] R. D'Andrea and G. E. Dullerud, "Distributed control design for spatially interconnected systems," IEEE Trans. on Automatic Control, vol. 48, no. 9, pp. 1478-1495, 2003.

[6] R. Cogill and S. Lall, "Control design for topology-independent stability of interconnected systems," in Proc. of the IEEE American Control Conference, Boston, 2004, pp. 3717 - 3722.

[7] Y. Liu, K. Passino, and M. Polycarpou, "Stability analysis of Mdimensional asynchronous swarms with fixed communication topology," IEEE Trans. on Automatic Control, vol. 48, no. 1, pp. 76-95, 2003.

[8] P. Chandler, S. Rasmussen, and M. Pachter, "UAV cooperative path planning," in Proc. of the AIAA Guidance, Navigation, and Control Conference and Exhibit, Denver, CO, 2000.

[9] M. Polycarpou, Y. Yang, and K. Passino, "Cooperative search framework for distributed agents," in Proc. of the 2001 IEEE International Symposium on Intelligent Control, Mexico City, Mexico, 2001, pp. 1-6.

[10] Y. L. Y. Jin, A. Minai, and M. Polycarpou, "Balancing search and target response in cooperative unmanned aerial vehicle (UAV) teams," IEEE Transaction on Systems, Man and Cybernetics: B, vol. 36, pp. 571-587, 2006.

[11] G. Grimm, M. Messina, S. Tuna, and A. Teel, "Model predictive control: for want of a local control Lyapunov function, all is not lost," IEEE Transaction on Automatic Control, vol. 50, pp. 546-558, 2005.

[12] — "Nominally robust model predictive control with state constraints," in Proc. of the IEEE Conf. on Decision and Control, Mauii, HI, 2003, pp. $1413-1418$.

[13] D. Limón, T. Alamo, F. Salas, and E. F. Camacho, "Input to state stability of min-max MPC controllers for nonlinear systems with bounded uncertainties," Automatica, vol. 42, pp. 797-803, 2006.

[14] W. B. Dunbar and R. M. Murray, "Distributed receding horizon control with application to multi-vehicle formation stabilization," Automatica, vol. 42, no. 4, pp. 549-558, 2006.

[15] W. Li and C. G. Cassandras, "Stability properties of a receding horizon controller for cooperating UAV's," in Proc. of the IEEE Conf. on Decision and Control, Bahamas, 2004, pp. 2905-2910.

[16] L. Magni and R. Scattolini, "Model predictive control of continuoustime nonlinear systems with piecewise constant control," IEEE Trans. on Automatic Control, vol. 49, pp. 900-906, 2004.

[17] L. Magni, D. M. Raimondo, and R. Scattolini, "Regional input-tostate stability for nonlinear model predictive control," IEEE Trans. on Automatic Control, vol. 51, no. 9, 2006.

[18] D. Mayne, J. Rawlings, C. Rao, and P. Skokaert, "Constrained model predictive control: Stability and optimality," Automatica, vol. 36, pp. 789-814, 2000

[19] W. Li and C. G. Cassandras, "A receding horizon approach for solving some cooperative control problems," in Proc. of the IEEE Conf. on Decision and Control, Las Vegas, 2002, pp. 3760-3765.

[20] — - "Stability properties of a cooperative receding horizon controller," in Proc. of the IEEE Conf. on Decision and Control, Hawaii, 2003, pp. 492-497.

[21] T. Kevicky, F. Borrelli, and G. J. Balas, "Hierarchical design of decentralized receding horizon controllers for decoupled systems," in Proc. of the IEEE Conf. on Decision and Control, Bahamas, 2004, pp. 1592 1597.
[22] _ _ "A study on decentralized receding horizon control for decoupled systems," in Proc. of the IEEE American Control Conference, Boston, 2004, pp. 4921-4926.

[23] W. B. Dunbar and R. M. Murray, "Receding horizon control of multivehicle formation: a distributed implementation," in Proc. of the IEEE Conf. on Decision and Control, Bahamas, 2004, pp. 1995-2002.

[24] H. G. Tanner, G. J. Pappas, and V. Kumar, "Input-to-state stability on formation graphs," in Proc. of the IEEE Conf. on Decision and Control, Las Vegas, 2002, pp. 2439-2444.

[25] — , "Leader to formation stability," IEEE Transactions on Robotics and Automation, vol. 20, no. 3, pp. 2129-2136, 2004.

[26] X. Chen and A. Serrani, "Remarks on ISS and formation control," in Proc. of the IEEE Conf. on Decision and Control, Bahamas, 2004, pp. 177-182.

[27] D. Nesic and A. R. Teel, "Input-output stability properties of networked control systems," IEEE Trans. on Automatic Control, vol. 49, no. 10, pp. 1650-1667, 2004.

[28] - "Input to state stability properties of networked control systems," Automatica, vol. 40, no. 12, pp. 2121-2128, 2004.

[29] E. Franco, T. Parisini, and M. Polycarpou, "Cooperative control of discrete-time agents with delayed information exchange: a recedinghorizon approach," in Proc. of the IEEE Conf. on Decision and Control, Bahamas, 2004, pp. 4274-4279.

[30] Z. P. Jiang and Y. Wang, "Input-to-state stability for discrete-time nonlinear systems," Automatica, vol. 37, no. 6, pp. 857-869, 2001.

[31] L. Magni, G. De Nicolao, L. Magnani, and R. Scattolini, "A stabilizing model-based predictive control algorithm for nonlinear systems," Automatica, vol. 37, no. 9, pp. 1351-1362, 2001

[32] E. D. Sontag and Y. Wang, "New characterizations of input-to statestability," IEEE Trans. on Automatic Control, vol. 41, pp. 1283-1294, 1996.

[33] K. Gao and Y. Lin, "On equivalent notions of input-to-state stability for nonlinear discrete time systems," in IASTED International Conference on Control and Applications, Cancun, Mexico, May 2000, pp. 81-86.

[34] H. Khalil, Nonlinear Systems. Prentice Hall, 2001.

[35] S. Dashkovskiy, B. Rüffer, and F. Wirth, "A small-gain type stability criterion for large scale networks of ISS systems," in Proc. of the IEEE Conf. on Decision and Control and European Control Conference, Seville, Spain, 2005, pp. 5633-5638.

[36] E. Franco, T. Parisini, and M. Polycarpou, "Design and stability analysis of cooperative receding-horizon control of linear discrete-time agents," Int. Journal on Robust and Nonlinear Control, 2006 (to appear).

[37] Z. Jin, S. Waydo, E. B. Wildanger, M. Lammers, H. Scholze, P. Foley, D. Held, and R. M. Murray, "The second generation Caltech multivehicle wireless testbed," in Proc. of the IEEE American Control Conference, Boston, 2004, pp. 5321 - 5326.

[38] D. Nesic and A. R. Teel, "A framework for stabilization of nonlinear sampled-data systems based on their approximate discrete time models," IEEE Trans. on Automatic Control, vol. 49, no. 7, pp. 1103-1122, 2004.

[39] D. Nesic, A. R. Teel, and E. D. Sontag, "Formulas relating $\mathcal{K} \mathcal{L}$ estimates of discrete time and sampled data nonlinear systems," Systems and Control Letters, vol. 38, no. 1, pp. 49-60, 1999.

[40] D. Nesic, A. R. Teel, and P. Kokotovic, "Sufficient condition for sampled-data stabilization of nonlinear systems via discrete time approximations," Systems and Control Letters, vol. 38, no. 4-5, pp. 259-270, 1999.

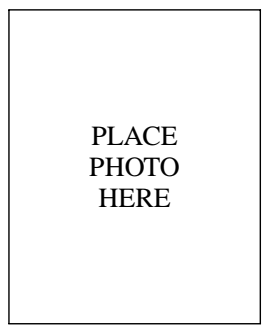

Elisa Franco Elisa Franco was born in Gorizia, Italy, in 1978. After graduating with full marks (summa cum laude) in Electrical Engineering at the University of Trieste in 2003, she got her PhD from the same institution in 2007. Currently she is pursuing a second doctorate degree at the California Institute of Technology in the Control and Dynamical Systems department, with a focus on synthetic biology. She was a visiting scholar in the Department of Mechanical and Aerospace Engineering at UCLA from April 2004 until May 2005. Her interests include Cooperative Control, Receding Horizon Control, Fault Diagnosis, System Identification, Design and Engineering of Biosynthetic Networks. 


\begin{tabular}{|c|}
\hline \\
PLACE \\
PHOTO \\
HERE \\
\end{tabular}

Lalo Magni Lalo Magni was born in Bormio (SO), Italy, in 1971. He graduated with full marks and honours (summa cum laude) in Computer Engineering at the University of Pavia, Italy, in 1994. He got his $\mathrm{PhD}$ in Electronic and Computer Engineering in 1998. From January 1st 1999 to December 31st 2004 he was Assistant Professor at the University of Pavia where he has been Associate Professor since January 1st 2005 . From October 1996 to February 1997 and in March 1998 he was at CESAME, Universitè Catholique de Louvain, Louvain La Neuve (Belgium). From October to November 1997 he was at the University of Twente with the System and Control Group in the Faculty of Applied Mathematics. In 2003 he was a plenary speaker at the 2nd IFAC Conference "CONTROL SYSTEMS DESIGN" (CSD’03). In 2005 he was Keynote speaker at the NMPC Workshop on Assessment and Future Direction. His current research interests include nonlinear control, predictive control, receding-horizon control, robust control, process control and glucose concentration control in subjects with diabetes. His research is witnessed by more than 30 papers published in the main international journals. In 2003, he was Guest Editor of the Special Issue "Control of nonlinear systems with Model Predictive Control" in the International Journal of Robust and Nonlinear Control. He served as an Associate Editor of the IEEE Transactions on Automatic Control. He is subarea Chair for the area "Nonlinear systems optimal and predictive control" at the IFAC Symposium on Nonlinear Control Systems (NOLCOS 2007). He will organize the NMPC Workshop on Assessment and Future Direction in September 2008 in Pavia.

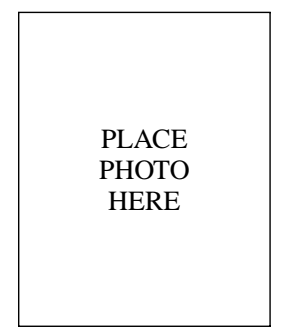

Thomas Parisini was born in Genoa, Italy, in 1963. He received the "Laurea" degree (Cum Laude and printing honours) in Electronic Engineering from the University of Genoa in 1988 and the Ph.D. degree in Electronic Engineering and Computer Science in 1993. From 1988 to 1995 , he was with the Dept. of Communications, Computer and Systems Sciences (DIST), University of Genoa. In 1995, he joined the Dept. of Electrical, Electronic and Computer Engineering (DEEI), University of Trieste, as an assistant professor, and in 1998, he joined the Dept. of Electronic and Information Engineering (DEI), Politecnico di Milano, as associate professor. In 2001 he was appointed full professor and Danieli Endowed Chair of Automation Engineering at the Dept. of Electrical, Electronic and Computer Engineering (DEEI), University of Trieste. Thomas Parisini is the present Chair of the IEEE Control Systems Society Conference Editorial Board. He was the Chair of the Technical Committee on Intelligent Control and he is an appointed member of the Board of Governors of the IEEE Control Systems Society. He is a Distinguished Lecturer of the IEEE Control Systems Society. He is the co-recipient of the 2004 Outstanding Paper Award of the IEEE Trans. on Neural Networks. Thomas Parisini is currently serving as an Associate Editor of Automatica, of the Int. J. of Control, and as Subject Editor of the Int. J. of Robust and Nonlinear Control and served as Associate Editor of the IEEE Trans. on Automatic Control, of the IEEE Trans. on Neural Networks, and as Subject Editor of the Int. J. of Adaptive Control and Signal Processing. He was a Guest Editor of several special issues in international journals including two special issues in the IEEE Trans. on Neural Networks, namely the special issue on Adaptive Learning Systems in Communication Networks and the special issue on Neural Networks for Feedback Control He was involved in the organization and in the technical program committees of several IEEE CSS sponsored conferences including the IEEE Conf. on Decision and Control and the IEEE Conf. on Control Applications. In particular, he was Vice-Program Chair of the 2003 IEEE Conf. on Decision and Control, 2003, the Program Chair of the IEEE Int. Symp. on Intelligent Control, held in Mexico City, 2001 and the Program Chair of the Joint IEEE Int. Symp. on Intelligent Control and Mediterrean Control Conference held in Limassol, Cyprus, June 2005. He was recently appointed as Program Chair of the 47th IEEE Conf. on Decision and Control to be held in Cancun, MX, in 2008 His research interests include neural-network approximations for optimal control and filtering problems, fault diagnosis for nonlinear systems, hybrid control systems and control of distributed systems. From an application point of view, he is involved as Project Leader in several projects funded by the European Union, by the Italian Ministry for Research and by some major process control companies (ABB, Danieli, Duferco, Galileo Avionics among others).

Marios M. Polycarpou Marios M. Polycarpou received the B.A. degree in Computer Science and the B.Sc. degree in Electrical Engineering both from Rice University, Houston, TX, USA in 1987, and the

PLACE PHOTO HERE M.S. and Ph.D. degrees in Electrical Engineering from the University of Southern California, Los Angeles, CA, in 1989 and 1992 respectively. In 1992, he joined the University of Cincinnati, Ohio, USA, where he reached the rank of Professor of Electrical and Computer Engineering and Computer Science. In 2001, he was the first faculty to join the newly established Department of Electrical and Computer Engineering at the University of Cyprus, where he is currently Professor and Dept. Chair. His teaching and research interests are in intelligent systems and control, adaptive and cooperative control systems, computational intelligence, fault diagnosis and distributed agents. He has published more than 160 refereed papers in these areas and one textbook, published by Wiley in 2006. He is also the holder of 3 patents. His research has been funded by several agencies in the United States, the European Commission and the Research Promotion Foundation of Cyprus. Marios Polycarpou is currently the Editor-in-Chief of the IEEE Transactions on Neural Networks. He is also an Associate Editor of two international journals and past Associate Editor of the IEEE Transactions on Neural Networks (1998-2003) and of the IEEE Transactions on Automatic Control (1999-2002). He was the recipient of the William H. Middendorf Research Excellence Award at the University of Cincinnati (1997) and was nominated by students for the Professor of the Year award (1996). He served as the Chair of the Technical Committee on Intelligent Control, IEEE Control Systems Society (2003-05) and as Vice President, Conferences, of the IEEE Computational Intelligence Society (2002-03). He is currently an appointed member of the Board of Governors of the IEEE Control Systems Society. $\mathrm{He}$ served as General Chair of the joint 2005 IEEE International Symposium on Intelligent Control and 2005 Mediterranean Conference on Control and Automation, and is currently General co-Chair of the 2007 International Symposium on Neural Networks. He is a Fellow of IEEE.

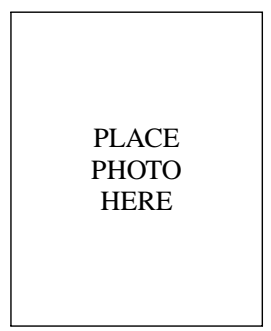

Davide Raimondo Davide Raimondo was born in Pavia, Italy in 1981. He received the Laurea degree (summa cum laude) in Computer Engineering from the Università di Pavia, Italy, in 2005. He is presently $\mathrm{Ph} . \mathrm{D}$. student in Electronic, Computer and Electric Engineering at the Università di Pavia, Italy. During November 2006 to May 2007 he was at the Universidad de Sevilla, Escuela Superior de Ingenieros. $\mathrm{He}$ was co-organizer of the invited session New Developments in Nonlinear Model Predictive Control for the IFAC Symposium on Nonlinear Control Systems (NOLCOS 2007). His current research interests include nonlinear control, predictive control, receding-horizon control, robust control, input-tostate stability, process control and glucose concentration control in subjects with diabetes. 